JOURNAL OF THE AMERICAN MATHEMATICAL SOCIETY

Volume 21, Number 2, April 2008, Pages 563-596

S 0894-0347(07)00558-9

Article electronically published on February 2, 2007

\title{
GROUPS, MEASURES, AND THE NIP
}

\author{
EHUD HRUSHOVSKI, YA'ACOV PETERZIL, AND ANAND PILLAY
}

\section{INTRODUCTION}

One of the occasions for writing this paper is the completion of the proof of the "o-minimal group conjectures" of the third author, from [23. Among the new ingredients are (i) the use of invariant measures on definable sets in the presence of the NIP (failure of the independence property) and (ii) the identification of a certain property (finitely satisfiable generics) which can be used in an inductive proof and is of interest in its own right.

The measures appear in Keisler's paper 13 which is a strong influence on our work. In Keisler's work, the theory of forking is in a sense extended from stable theories to theories without the independence property, but replacing complete types by measures (on the Boolean algebra of definable sets). It is somewhat amusing to note that Keisler's work was roughly contemporaneous with early work on $o$-minimality which was also motivated by the attempt to generalize stability to suitable ordered structures.

Our work may also overlap to some extent with recent papers of Shelah on theories without the independence property (for example [26], 27]).

In any case, we take the opportunity in this paper to expand on and develop some theory, not all of which is directed towards the proof of the $o$-minimal group conjectures.

Stability and stable group theory are at the core of "pure" or "abstract" model theory. Recall Shelah's result that $T$ is stable iff $T$ does not have the strict order property and does not have the independence property (see [26]). There has been considerable work on generalizing stability to particularly nice theories without the strict order property, namely the simple theories. So part of this paper surrounds developing some theory in an "orthogonal" direction, namely for certain theories $T$ without the independence property. Another aspect of this paper is the "model theory of the standard part map".

In Section 2, we recall and elaborate on some of Keisler's notions from [13. In particular we discuss smooth, definable, and finitely satisfiable measures. In Section 3, we discuss some consequences of NIP, sometimes in the presence of measures. Included here is a "Borel definability" of coheirs assuming NIP. In Section 4, we

Received by the editors July 16, 2006.

2000 Mathematics Subject Classification. Primary 03C68, 03C45, 22C05, 28 E05.

Key words and phrases. o-minimal, independence property, compact Lie group, Keisler measure.

The first author was supported by the Israel Science Foundation grant no. 244/03.

The last author was supported by NSF grants DMS-0300639 and FRG DMS-0100979, as well as a Marie Curie chair. 
introduce the "finitely satisfiable generics" property for definable groups $G$, stating which aspects of stable group theory are valid in this situation. In Section 5 we discuss in general "definably amenable groups", namely groups with a left invariant measure on the definable sets. In Section 6 we prove various results surrounding existence of $G^{00}$ and existence of invariant measures under the NIP assumption. In Section 7 we take a short diversion to explain how our results can generalize to the class of "inductively definable" groups. In Section 8 we prove the full conjecture from [23]:

(*) If $G$ is a definably compact group definable in a saturated o-minimal expansion of a real closed field, then the quotient $G / G^{00}$ of $G$ by its smallest type-definable subgroup of bounded index $G^{00}$ is, when equipped with the logic topology, a compact Lie group whose dimension (as a Lie group) equals the dimension of $G$ (as a definable set in an o-minimal structure).

The proof rests on and continues a number of earlier papers [23], [2, [4], 19], and [7]. We will give below a guide for the reader who is interested in a fast path to the proof of $(*)$.

In Section 9 and 10, we isolate a new notion, of "compact domination", and conjecture that in fact a definably compact group $G$ in an $o$-minimal structure is compactly dominated by $G / G^{00}$. We then prove this in several special cases.

Guide to the proof of $\left({ }^{*}\right)$. The proof is carried out in Section 8. Globally it proceeds by induction on $\operatorname{dim}(G)$. The two extreme cases are when (a) $G$ is commutative and (b) $G$ is definably simple. The "new" ingredient for case (a) is use of the amenability of $G$ (namely the existence of an invariant finitely additive measure on all subsets of $G$ ) together with the NIP. The key sequence of preliminary results is Lemma 2.8, Proposition 3.3, Corollary 3.4 and Proposition 6.3. Case (a) is proved in Lemma 8.2. Case (b) was proved in [19] under the weaker hypothesis that " $G$ has very good reduction". This is discussed in Lemma 8.3 of the current paper. For the induction step, one may assume $G$ has a normal commutative definable subgroup $N$. But we need to know more than simply that $(*)$ holds for $G / N$ and $N$. Namely we require that both $G / N$ and $N$ have the "finitely satisfiable generics" property. The $f s g$ is introduced in Section 4, and Proposition 4.2 is crucial. In Cases (a) and (b) we actually prove in addition that the relevant groups have the $f s g$ property. Proposition 4.5 shows that from the $f s g$ for $G / N$ and $N$ we can conclude the $f s g$ for $G$. An argument using Corollary 4.3 shows that $\left(^{*}\right)$ holds for $G$.

Our notation is standard. We work in a large saturated model $\bar{M}$ of a complete first order, possibly many-sorted theory $T$ in a language $L$. If we assume that $|\bar{M}|=\bar{\kappa}$, then by a "small" or "bounded" set we mean a set of cardinality $<\bar{\kappa}$. We let $x, y$ denote finite sequences of variables unless we say otherwise. $A, B, \ldots$ denote small subsets of $\bar{M}$, and $M, N, \ldots$ denote small elementary substructures of $\bar{M}$. "Type-definable" means the intersection of a small collection of definable sets, and a "bounded type-definable equivalence relation" is a type-definable equivalence relation with a bounded number of classes. We refer to [24] for any background on stability.

$T$ is said to have the NIP (for "not the independence property") if there is no formula $\phi(x, y) \in L$ and $\left\langle a_{i}: i<\omega\right\rangle$ and $\left\langle b_{w}: w \subseteq \omega\right\rangle$ such that $\models \phi\left(a_{i}, b_{w}\right)$ iff $i \in \omega$. Stable and $o$-minimal theories, as well as the theory of the $p$-adic field, are all examples of theories with NIP, while simple unstable theories all have the independence property. 
If $G$ is a group definable in $\bar{M}$, then $G^{00}$ is the smallest type-definable subgroup of bounded index in $G$, if there is such. If $E$ is a type-definable equivalence relation on a definable set $X$ with a bounded number of classes, then the logic topology on $X / E$ is given by: $C \subseteq X / E$ is closed if the pre-image of $C$ in $X$ is type-definable.

In various parts of the paper we will make use of standard facts and techniques regarding indiscernibles, which the referee has asked us to explain. One of these facts is that given a complete theory $T$ and cardinal $\mu$ there is a cardinal $\lambda$ such that if $\left\{a_{\alpha}: \alpha<\lambda\right\}$ is a set of $\mu$-tuples in some saturated model of $T$, then there is an indiscernible sequence $\left(b_{i}: i<\omega\right)$ of $\mu$-tuples, such that for every $n, t p\left(b_{0}, \ldots, b_{n-1}\right)=t p\left(a_{\alpha_{0}}, \ldots, a_{\alpha_{n-1}}\right)$ for some $\alpha_{0}<\ldots<\alpha_{n-1}<\lambda$. This is an application of the Erdös-Rado Theorem. A statement and proof appear in [10] (Theorem 1.13) for example. When using this fact, we will just say "by Erdös-Rado". Another method is "stretching" indiscernibles: namely, given an indiscernible sequence $\left(a_{i}: i<\omega\right)$, we can, for any totally ordered set $I$, find an indiscernible sequence $\left(b_{i}: i \in I\right)$ such that for each $n$ and $i_{0}<\ldots<i_{n}$ in $I$, $t p\left(b_{i_{0}}, \ldots, b_{i_{n-1}}\right)=t p\left(a_{0}, \ldots, a_{n-1}\right)$. This is of course just by compactness.

\section{Definable functions and measures}

We consider here functions of one kind or another from sorts, or definable sets in $\bar{M}$, to compact Hausdorff spaces $C$, such as the closed interval $[0,1]$.

Definition 2.1. Let $X$ be an $A$-definable set in $\bar{M}, C$ some compact Hausdorff space of bounded size, and $f$ a map from $X$ to $C$. We will say that $f$ is definable over $A$ if for any closed subset $C_{1}$ of $C, f^{-1}\left(C_{1}\right) \subseteq X$ is type-definable over $A$ in $M$.

Example 2.2. (i) The tautological map $s$ from $X$ to its Stone space $S_{X}(A): s(b)=$ $t p(b / A)$. Note that a map $f$ from $X$ to a compact Hausdorff space $C$ will be definable over $A$ just if $f=g \circ s$ with $g$ a continuous map from $S_{X}(A)$ to $C$. So the tautological definable map $s$ is also universal.

(ii) Let $A$ be a small subset of sort $X$ in $\bar{M}$, and let $\phi(x, y)$ be a formula with $x$ of sort $X$ and $y$ of sort $Y$. Identify the power set of $A$ with the compact space $2^{|A|}$. Let $f: Y \rightarrow 2^{|A|}$ be given by $f(b)=\{a \in A: \mid=\phi(a, b)\}$. Then, as is easy to verify, $f$ is definable over $A$.

In Definition 2.1, note that if $f: X \rightarrow C$ is definable, then $f(X) \subseteq C$ is closed (because as in Example 2.2(i), $f$ can be identified with a continuous map between compact spaces; hence its image is closed). So we may assume $f$ to be onto.

In fact definable maps as in Definition 2.1 amount to the same thing as quotienting by bounded type-definable equivalence relations:

Remark 2.3. Let $X$ be definable over $A$ in $\bar{M}$.

(i) Let $f$ be a definable (over $A$ ) map from $X$ onto the compact Hausdorff space $C$ in the sense of Definition 2.1. Let $E=\{(x, y) \in X \times X: f(x)=f(y)\}$. Then $E$ is an $A$-type-definable equivalence relation of bounded index, and $f$ induces a homeomorphism between $X / E$ with the logic topology and the space $C$.

(ii) Conversely, if $E$ is a bounded $A$-type-definable equivalence relation on $X$, $X / E$ is equipped with the logic topology, and $M_{0}$ is a small model containing $A$ and a representative for each $E$-class, then the quotient map $f: X \rightarrow X / E$ is an $M_{0}$-definable map from $X$ onto the compact Hausdorff space $X / E$. 
Proof. (i) For each pair $C_{1}, C_{2}$ of closed subsets of $C$ such that $C_{1} \cup C_{2}=C$, let $E_{C_{1}, C_{2}}=\left\{(x, y) \in X \times X\right.$ such that either $f(x) \in C_{1}$ and $f(y) \in C_{1}$ or $f(x) \in C_{2}$ and $\left.f(y) \in C_{2}\right\}$. So $E_{C_{1}, C_{2}}$ is type-definable over $A$. As $X$ is Hausdorff, $E$ is the intersection of all $E_{C_{1}, C_{2}}$ and hence is also type-definable. Identifying $X / E$ with $C$, we see that the logic topology on $C$ refines the original topology on $C$. As both topologies are compact Hausdorff, they agree. $E$ is of bounded index since the pre-image of each singleton in $C$ is type-definable over a fixed set $A$.

(ii) If $C \subseteq X / E$ is closed, then by definition $f^{-1}(C)$ is type-definable. But $f^{-1}(C)$ is also $M_{0}$-invariant; hence it is type-definable over $M_{0}$.

So Definition 2.1 is cosmetic. However it enables some unification of various notions, as well as some clean statements. For example the conjecture from 23 can now be restated:

If $G$ is a definably connected definably compact group in a saturated o-minimal structure $M$, then there is a definable surjective homomorphism $f$ from $G$ to a compact Lie group $G_{1}$ where $\operatorname{dim}\left(G_{1}\right)$ equals the o-minimal dimension of $G$. Moreover any other definable homomorphism from $G$ into a compact group factors through $f$.

We now recall the probability measures on definable sets considered by Keisler 13. We will call these Keisler measures. Let us fix again a sort or definable set $X$ in $\bar{M}$ which we assume to be $\emptyset$-definable. $\operatorname{Def}(X)$ will denote the subsets of $X$ definable (with parameters) in $\bar{M}$, and $D e f_{A}(X)$ those sets defined over $A$. (So we identify $\operatorname{Def}(X)$ with $\operatorname{Def} f_{\bar{M}}(X)$.)

Definition 2.4. (i) A Keisler measure $\mu$ on $X$ over $A$ is a finitely additive probability measure on $\operatorname{Def}_{A}(X)$; namely a map $\mu$ from $D e f_{A}(X)$ to the interval $[0,1]$ such that $\mu(\emptyset)=0, \mu(X)=1$ and for $Y, Z \in \operatorname{Def}_{A}(X), \mu(Y \cup Z)=$ $\mu(Y)+\mu(Z)-\mu(Y \cap Z)$.

(ii) $A$ (global) Keisler measure on $X$ is a finitely additive probability measure on $\operatorname{Def}(X)$.

(iii) If $\mu$ is a Keisler measure on $D e f_{B}(X)$ and $A \subseteq B$, we write $\mu \mid A$ for the restriction of $\mu$ to $\operatorname{Def}_{A}(X)$.

Note that a complete type (of an element of $X$ ) over $A$ is precisely a $0-1$ valued Keisler measure on $X$ over $A$.

For each $L$-formula $\phi(x, y)$ with $x$ a variable of sort $X$, let $S_{\phi}$ be the sort whose elements are the subsets of $X$ defined by instances of $\phi$. So a global Keisler measure on $X$ is given through a family $\left\{\mu_{\phi}: \phi(x, y) \in L\right\}$ of maps $\mu_{\phi}: S_{\phi} \rightarrow[0,1]$.

Keisler observes that any Keisler measure on $X$ over $A$ extends to a global Keisler measure on $X$. Moreover any Keisler measure on $X$ over $A$ extends to a unique countably additive measure on the $\sigma$-algebra generated by the $A$-definable subsets of $X$ such that the measure of an open set $U$ is the supremum of the measures of the subsets of $U$ which are definable (over $A$ ) (see Theorem 1.2 in 13). In fact this Borel measure will be regular. We will point out now a way of extending a Keisler measure over a model to a global Keisler measure, as the construction will be useful later on.

Construction (*). Let $\mu$ be a Keisler measure on $X$ over a model $M_{0}$, viewed as a map from definable in $M_{0}$ subsets of $X\left(M_{0}\right)$ to [0,1]. Consider the structure $\left\langle M_{0},[0,1],+,<, \mu_{\phi}\right\rangle_{\phi}$ consisting of $M_{0}^{e q}$, the real unit interval $[0,1]$, and for each $\phi$, the map $\mu_{\phi}: S_{\phi}\left(M_{0}\right) \rightarrow[0,1]$ as well as the ordering and addition (modulo 1 ) on $[0,1]$. Take a saturated elementary extension $\left\langle M_{0}^{\prime},[0,1]^{\prime},+,<, \mu_{\phi}^{\prime}\right\rangle_{\phi}$. Then the 
composition of $\mu^{\prime}$ with the standard part map st $:[0,1]^{\prime} \rightarrow[0,1]$ is a Keisler measure on $X$ over $M_{0}^{\prime}$ extending $\mu$. We may identify $\bar{M}$ with $M_{0}^{\prime}$.

One point of this construction is that the structure $\bar{M}$, equipped with the constructed measure, has some obvious "saturation" properties.

We have observed that a Keisler measure on $X$ is (among other things) a sequence of maps from sorts $S_{\phi}$ to $[0,1]$. It would be natural to call $\mu$ definable if each $\mu_{\phi}: S_{\phi} \rightarrow[0,1]$ is definable in the sense of Definition 2.1. This is precisely (i) in the next definition.

Definition 2.5. Let $\mu$ be a (global) Keisler measure on $X$.

(i) Then $\mu$ is definable over $A$ iff for each $L$-formula $\phi(x, y)$ and closed subset $C$ of $[0,1],\{b \in M: \mu(\phi(x, b)) \in C\}$ is type-definable over $A$.

Let $M_{0}$ be a small submodel of $\bar{M}$.

(ii) We say that $\mu$ is finitely satisfiable in $M_{0}$ if whenever $Y \subseteq X$ is definable and $\mu(Y)>0$, then $Y \cap M_{0} \neq \emptyset$.

(iii) We say that $\mu$ is smooth over $M_{0}$ if $\mu$ is the unique (global) extension of $\mu \mid M_{0}$ to a measure on $X$. In this situation we also say that $\mu \mid M_{0}$ is smooth.

The notion of a smooth measure was also introduced by Keisler ([13]) although his definition is weaker than the above, for certain technical reasons. In any case, if $\mu$ is a $0-1$ measure given by a complete type, then it is smooth if and only if the type is algebraic.

Here is a "nonalgebraic" example of a smooth Keisler measure: Let $\bar{M}$ be a saturated real closed field, and take $X$ to be the interval $[0,1]$ in the sense of $\bar{M}$. The field of reals $\mathbb{R}$ is an elementary substructure of $\bar{M}$. The standard measure on the real unit interval $[0,1]^{\mathbb{R}}$ gives a Keisler measure on $X$ over $\mathbb{R}$ which is easily seen to have a unique extension over $\bar{M}$. (This will be subsequently generalized in the last section.)

Lemma 2.6. Let $\mu$ be a (global) Keisler measure on $X$. Suppose that $\mu$ is smooth over $M_{0}$. Then $\mu$ is both finitely satisfiable in $M_{0}$ and definable over $M_{0}$.

Proof. Finite satisfiability is immediate from [13], Lemma 2.2 (which is itself based on Lemma 1.6 there), but for the sake of completeness we repeat the argument here.

It is clearly sufficient to prove that if $X$ is a definable set in $\bar{M}$ with $\mu(X)>0$, then it contains an $M_{0}$-definable $Y$ with $\mu(Y)>0$. Assume not, namely that all $M_{0}$-definable subsets of $X$ have $\mu$-measure zero. By the smoothness assumption, it is sufficient to show that there is some finitely additive Keisler measure $\mu^{\prime}$ on $\bar{M}$, extending $\mu \mid M_{0}$, with $\mu^{\prime}(X)=0$. By compactness, this amounts to showing, given finitely many $M_{0}$-definable sets $Y_{1}, \ldots, Y_{k}$, that there is a finitely additive probability measure $\mu^{\prime}$ on the Boolean algebra generated by $Y_{1}, \ldots, Y_{k}, X$, which agrees with $\mu$ on the $Y_{i}$ 's. Let $\mathcal{B}_{0}$ be the Boolean algebra generated by the $Y_{i}$ 's. Without loss of generality, the $Y_{i}$ 's are atoms in $\mathcal{B}_{0}$ and hence each $Y_{i} \cap X$ is an atom in the Boolean algebra generated by $\mathcal{B}_{0}$ and $X$. We now let $\mu^{\prime}(Y)=\mu(Y)$ for all $Y \in \mathcal{B}_{0}$ and $\mu^{\prime}\left(Y_{i} \cap X\right)=0$. This gives the desired measure $\mu^{\prime}$ and proves that $\mu$ is finitely satisfiable.

The definability of $\mu$ over $M_{0}$ is more or less explained by a "Beth's Theorem for continuous logic". But we will be more direct. We make use of Construction $(*)$ above. Consider the structure $\left\langle M_{0},[0,1],+,<, \mu_{\phi} \mid M_{0}\right\rangle_{\phi}$ from there, equipped with constants for all elements (of $M_{0}$ and of the unit interval). Let $T_{1}$ be its theory. 
We saw that in a saturated model $\bar{M}_{1}$ of $T_{1},\left\{s t \circ \mu_{\phi}^{\prime}: \phi \in L\right\}$ gives rise to a Keisler measure $\mu^{\prime \prime}$ extending $\mu \mid M_{0}$. We may assume that $\bar{M}_{1}$ is an expansion of $\bar{M}$, and by the smoothness assumption, that $\mu^{\prime \prime}=\mu$.

Fix an $L$-formula $\phi(x, y)$ where $x$ is of sort $X$. Given a closed set $C \subseteq[0,1]$, we want to show that the set $X_{1}=\{b: \mu(\phi(x, b)) \in C\}$ is type-definable in $\bar{M}$ over $M_{0}$. Note that the standard part map st $:[0,1]^{\prime} \rightarrow[0,1]$ (where $[0,1]^{\prime}$ is the unit interval in $\bar{M}_{1}$ ) is definable in $\bar{M}_{1}$ (over the empty set) in the sense of Definition 2.1. and by the definability of $\mu$ in $\bar{M}_{1}$, the set $X_{1}$ is type-definable over $M_{0}$ in $\bar{M}_{1}$, via a type $\Sigma(y)$.

Now, the smoothness assumption implies that $\Sigma(y)$ does not depend on the particular expansion $\bar{M}_{1}$ of $\bar{M}$. We can now apply the classical Beth Theorem (for types rather than formulas) and conclude that $X_{1}$ is type-definable in $\bar{M}$, over $M_{0}$.

Remark 2.7. Let $\mu$ be a global Keisler measure on $X$. Let us define $\mu$ to be an heir of $\mu \mid M_{0}$ if for each $L$-formula $\phi(x, y)$ and $r \in[0,1)$, if for some $b \in \bar{M}, \mu(\phi(x, b))>r$, then for some $b \in M_{0}, \mu(\phi(x, b))>r$. Then the proof above shows that $\mu$ is the unique heir of $\mu \mid M_{0}$ over $\bar{M}$ if and only if $\mu$ is definable over $M_{0}$.

The following relationship between Keisler measures and indiscernibles will be useful. It also appears in [14].

Lemma 2.8. Let $\mu$ be a Keisler measure on $X$. Let $x$ be a variable of sort $X$, let $\phi(x, y) \in L$, and let $\left\langle b_{i}: i<\omega\right\rangle$ be an indiscernible sequence such that for some $\epsilon>0, \mu\left(\phi\left(x, b_{i}\right)\right) \geq \epsilon$ for all $i$. Then $\left\{\phi\left(x, b_{i}\right): i<\omega\right\}$ is consistent.

Proof. Let $Y_{b_{i}}$ denote the set defined by $\phi\left(x, b_{i}\right)$. By Construction $(*)$ above and Ramsey's theorem, we may assume that the sequence $\left\langle b_{i}: i\langle\omega\rangle\right.$ is also indiscernible with respect to the map $\mu$, in particular that for each $i_{1}<\ldots<i_{n}<\omega$ and $j_{1}<\ldots<j_{n}<\omega, \mu\left(Y_{b_{i_{1}}} \cap \ldots \cap Y_{b_{i_{n}}}\right)=\mu\left(Y_{b_{j_{1}}} \cap \ldots \cap Y_{b_{j_{n}}}\right)=r_{n}$ say. So by assumption, $r_{1}>0$.

Suppose for a contradiction that some finite intersection of the $Y_{b_{i}}$ 's is empty. Choose maximal $k$ such that $r_{k}>0$. For $j \geq 0$ let $Z_{j}=Y_{b_{1}} \cap Y_{b_{2}} \cap \ldots \cap Y_{b_{k-1}} \cap Y_{b_{k+j}}$. Then each $Z_{j}$ has measure $r_{k}>0$ and their pairwise intersections have measure 0 , a contradiction.

\section{NIP AND SOME CONSEQUENCES}

The definition of NIP (failure of independence property) was given in the Introduction. A well-known equivalence (see Theorem 12.17 of [25]) is

Lemma 3.1. $T$ has the NIP if and only if for any sequence $\left\langle b_{i}: i<\omega\right\rangle$ which is indiscernible over $\emptyset$ and formula $\phi(y)$, possibly with parameters, there is an $i$ such that $=\phi\left(b_{j}\right)$ for all $j>i$, or $=\neg \phi\left(b_{j}\right)$ for all $j<i$.

Notation: If $\phi(x), \psi(x)$ are formulas, let $\phi(x) \Delta \psi(x)$ denote the symmetric difference $(\phi(x) \wedge \neg \psi(x)) \vee(\neg \phi(x) \wedge \psi(x))$ of $\phi$ and $\psi$.

Corollary 3.2. Suppose $T$ has NIP. Let $\phi(x, y)$ be an L-formula and $\left\langle b_{i}: i<\omega\right\rangle$ an indiscernible sequence. Then the set $\left\{\phi\left(x, b_{2 j}\right) \Delta \phi\left(x, b_{2 j+1}\right): j<\omega\right\}$ is inconsistent.

Proof. Otherwise, let $c$ realize $\left\{\phi\left(x, b_{2 j}\right) \Delta \phi\left(x, b_{2 j+1}\right): j<\omega\right\}$ and the formula $\phi(c, y)$ contradicts Lemma 3.1 . 
We now give some consequences of the NIP for Keisler measures. The main insight is due to Keisler ([13], Theorem 3.14). We are back to the context of $\bar{M}$ a saturated model of $T$ and $X$ a sort or $\emptyset$-definable set in $\bar{M}$.

Proposition 3.3. Assume T has the NIP. Let $\mu$ be a (global) Keisler measure on $X$. Let $\phi(x, y)$ be a formula with $x$ of sort $X$, and let $\epsilon>0$. Then there do not exist $\left\langle b_{i}: i<\omega\right\rangle$ such that $i \neq j$ implies $\mu\left(\phi\left(x, b_{i}\right) \Delta \phi\left(x, b_{j}\right)\right) \geq \epsilon$.

Proof. Suppose otherwise. Then by Construction $(*)$ from Section 2 and Ramsey's theorem, we may assume in addition that $\left\langle b_{i}: i\langle\omega\rangle\right.$ is indiscernible. By Lemma 2.8. $\left\{\phi\left(x, b_{2 j}\right) \Delta \phi\left(x, b_{2 j+1}\right): j<\omega\right\}$ is consistent, contradicting Corollary 3.2 .

Corollary 3.4. Assume $T$ has NIP and let $\mu$ be a global Keisler measure on $X$. For definable subsets $Y, Z$ of $X$, define $Y \sim_{\mu} Z$ if $\mu(Y \Delta Z)=0$. Then there are only boundedly many $\sim_{\mu}$-classes of definable subsets of $X$. In particular there is a small model $M_{0}$ such that every definable subset $Y$ of $X$ is $\sim_{\mu}$ to some $M_{0}$-definable subset of $X$.

Proof. If there are unboundedly many definable subsets of $X$ modulo $\sim_{\mu}$, then we can clearly find a formula $\phi(x, y)$ and large set $\left\langle b_{i}: i \in I\right\rangle$ such that the measures of the pairwise symmetric differences of the $\phi\left(x, b_{i}\right)$ are $>0$. By Construction $\left(^{*}\right)$ from Section 2, we may assume that $\left\langle b_{i}: i \in I\right\rangle$ is an indiscernible sequence with respect to $\mu$ as well, whereby $\mu\left(\phi\left(x, b_{i}\right) \Delta \phi\left(x, b_{j}\right)\right) \geq \epsilon$ for some fixed $\epsilon>0$ and all $i \neq j$. This contradicts Proposition 3.3.

Our next result is in a somewhat different spirit.

Theorem 3.5. Suppose $T$ is countable with NIP. Let $M_{0}$ be a countable elementary substructure of $\bar{M}$. Let $p(x)$ be a complete 1-type over $\bar{M}$ which is finitely satisfiable in $M_{0}$. Let $U=\left\{X \cap M_{0}: X \in p\right\}$. Then $U$ is a Borel (in fact an $F_{\sigma}$ ) subset of the Polish space $2^{M_{0}}$.

Before going into the proof, we give an easy example to illustrate the technique.

Remark 3.6. Let $T$ be countable, and let $M_{0}$ be a countable model. Then the following hold.

(i) The set $\left\{X \cap M_{0}: X\right.$ a definable subset of $\left.\bar{M}\right\}$ is an $F_{\sigma}$ (as a subset of $2^{M_{0}}$ ).

(ii) Let $p(x) \in S_{1}(\bar{M})$ be definable. Then $\left\{X \cap M_{0}: X \in p\right\}$ is an $F_{\sigma}$.

Proof. (i) Fix an $L$-formula $\phi(x, y)$, and let $n<\omega$. Let $U_{\phi}=\left\{X \cap M_{0}: X\right.$ is defined by $\phi(x, c)$ for some $c\}$. By Example 2.2(ii), $U_{\phi}$ is closed. Then $U=\bigcup_{\phi} U_{\phi}$ is Borel and coincides with $\left\{X \cap M_{0}: X\right.$ a definable subset of $\left.\bar{M}\right\}$.

(ii) Suppose again $\phi(x, y) \in L$ and let $\psi(y, d)$ be a formula defining $p \mid \phi$. Then define $U_{\phi}$ just as above but requiring also that $c$ realizes $\psi(y, d)$.

The proof of Theorem 3.5 will go through several lemmas.

For now let $T$ be an arbitrary complete theory with NIP.

Lemma 3.7. For any $\phi(x, y) \in L$, there is some $N=N_{\phi}$, such that for any indiscernible sequence $\left\langle a_{i}: i<\omega\right\rangle$ and $c$, there do not exists $i_{0}<i_{1}<\ldots<i_{N}$ such that for each $j<N, \models \phi\left(a_{i_{j}}, c\right) \leftrightarrow \neg \phi\left(a_{i_{j+1}}, c\right)$.

Proof. Otherwise, by compactness we find an indiscernible sequence $\left\langle a_{i}: i<\omega\right\rangle$ and $c$ such that for each $i<\omega, \models \phi\left(a_{i}, c\right)$ iff $\models \neg \phi\left(a_{i+1}, c\right)$, contradicting Lemma 3.1 . 
Recall that a type $p(x) \in S(\bar{M})$ is called finitely satisfiable in a model $M_{0} \subseteq \bar{M}$ if every formula in $p(x)$ is satisfiable in $M_{0}$. If $p(x) \in S(\bar{M})$ is finitely satisfiable in a small model $M_{0}$, then we can build an indiscernible sequence $I=\left\langle a_{0}, a_{1}, \ldots\right\rangle$ over $M_{0}$ by letting $a_{0}$ realize $p \mid M_{0}$ and $a_{i+1}$ realize $p \mid\left(M_{0} \cup\left\{a_{0}, \ldots, a_{i}\right\}\right)$. Although the sequence $I$ is not unique, its type $\operatorname{tp}\left(\left\langle a_{i}: i<\omega\right\rangle / M_{0}\right)$ IS unique, and we call this type $Q_{p, M_{0}}$.

Let us now fix a type $p(x) \in S(\bar{M})$ which is finitely satisfiable in $M_{0}$, and let $Q=Q_{p, M_{0}}$. (So $Q$ is a complete type over $M_{0}$ in variables $\left(x_{i}: i<\omega\right)$ ). Let $Q_{n}$ be the restriction of $Q$ to the variables $\left(x_{0}, \ldots, x_{n}\right)$. Fix an $L$-formula $\phi(x, y)$ and some $c$ from $\bar{M}$. We will say that a realization $\left(a_{0}, \ldots, a_{n}\right)$ of $Q_{n}$ is $\operatorname{good}$ for $\phi(x, c)$, if

(i) $\models \phi\left(a_{i}, c\right) \leftrightarrow \neg \phi\left(a_{i+1}, c\right)$ for all $i<n$, and

(ii) there does not exist $a_{n+1}$ such that $\left(a_{0}, \ldots, a_{n}, a_{n+1}\right)$ realizes $Q_{n+1}$ and

$\models \phi\left(a_{n}, c\right) \leftrightarrow \neg \phi\left(a_{n+1}, c\right)$.

With this notation, we have the following:

Lemma 3.8. For $p$ as above, the following are equivalent:

(i) $\phi(x, c) \in p$,

(ii) there is $k \leq N_{\phi}$ and there is a realization $\left(a_{0}, \ldots, a_{k}\right)$ of $Q_{k}$ which is good for $\phi(x, c)$ such that $\models \phi\left(a_{k}, c\right)$,

Proof. Note first that by Lemma 3.7, for any $c$ there is $k \leq N_{\phi}$ and realization $\left(a_{0}, \ldots, a_{k}\right)$ of $Q_{k}$ which is good for $\phi(x, c)$.

Now suppose $\left(a_{0}, \ldots, a_{k}\right)$ realizes $Q_{k}$ and is good for $\phi(x, c)$. Let $M_{1}$ be a small model containing $M_{0} \cup\left\{a_{0}, \ldots, a_{k}, c\right\}$ and let $a$ realize $p \mid M_{1}$. Note that $\left(a_{0}, \ldots, a_{k}, a\right)$ realizes $Q_{k+1}$. By the "goodness" of $\left(a_{0}, \ldots, a_{k}\right)$ for $\phi(x, c)$, it follows that $=\phi\left(a_{k}, c\right) \leftrightarrow \phi(a, c)$. But $\models \phi(a, c)$ iff $\phi(x, c) \in p$.

This is enough to prove the lemma.

Let us now assume $T$ and $M_{0}$ to be countable. We introduce some more notation: Fix $k$, and let $\left(Q_{k}^{i}: i<\omega\right)$ be an enumeration of the formulas in $Q_{k}$. Let $\psi_{k}^{i}\left(x_{0}, \ldots, x_{k}, y\right)$ be the formula " $Q_{k}^{i}\left(x_{0}, \ldots, x_{k}\right) \wedge \bigwedge_{j<k}\left(\phi\left(x_{j}, y\right) \leftrightarrow \neg \phi\left(x_{j+1}, y\right)\right)$ ". Let $\chi_{k}^{j, i}(y)$ be

$$
\text { " } \exists x_{0}, \ldots, x_{k}\left(\psi_{k}^{j}\left(x_{0}, \ldots, x_{k}, y\right) \wedge\left(\neg \exists x_{k+1}\left(\psi_{k+1}^{i}\left(x_{0}, \ldots, x_{k+1}\right)\right)\right) \wedge \phi\left(x_{k+1}, y\right)\right) " .
$$

Corollary 3.9. For any $c \in \bar{M}, \phi(x, c) \in p$ if and only if there is $k \leq N_{\phi}$ and there is $i<\omega$ such that c satisfies the formula $\chi_{k}^{j, i}(y)$ for all $j<\omega$.

Proof. By Lemma 3.8 and the notation.

Note that Corollary 3.9 gives us an $F_{\sigma}$-definition for $p$ over $M_{0}$.

In any case Theorem 3.5 follows from Corollary 3.9 as in the proofs of Remark 3.6. Note that the only real assumption on $p$ we need is that it is finitely satisfiable in some small model (not necessarily $M_{0}$ ).

\section{Groups With Finitely SAtisfiable Generics}

Here we introduce a certain desirable property of definable groups which we call $f s g$ (standing for "finitely satisfiable generics"). In Section 7 of the paper we prove that definably compact groups definable in $o$-minimal expansions of real closed fields have $f s g$. 
Again we fix a saturated model $\bar{M}$ of $T$, and $G$ will denote a group, definable in $\bar{M}$ over $\emptyset$.

Definition 4.1. $G$ has $f s g$ (finitely satisfiable generics) if there is some global type $p(x)$ and some small model $M_{0}$ such that $p(x) \models$ " $x \in G$ ", and every left translate $g p=\left\{\phi(x): \phi\left(g^{-1} x\right) \in p\right\}$ of $p$ with $g \in G$ is finitely satisfiable in $M_{0}$.

The basic example of such a group is a stable group. (If $G$ is stable, then there exists a global generic type $p$ of $G$ in the sense of stable group theory, namely every translate of $p$ does not fork over $\emptyset$. But then by the characterization of forking in the stable context, every translate of $p$ is finitely satisfiable in any submodel $M_{0}$.) In simple theories, however, definable groups will not, as a rule, have $f s g$. Also, the ordered group $\langle\mathbb{R},<,+\rangle$ does not have $f s g$. On the other hand the generically metastable groups from [12] which were introduced in connection with definability in algebraically closed-valued fields $d o$ have $f s g$.

For the remainder of this paper we call a definable subset $X$ of $G$ (or the formula defining it) left generic if finitely many left translates of $X$ cover $G$ and likewise with right generic. $X$ is generic if it is both left and right generic. A partial type $\Sigma(x)$ implying $x \in G$ is left (right) generic if every formula in $\Sigma(x)$ is. Although this is in accordance with established vocabulary in the case of stable theories, one should be aware that there is a discrepancy in the case of simple theories. Notice that if $p$ is a global type in $G$ and if $X$ is a definable left generic subset of $G$, then some left translate of $X$ (i.e. of the formula " $x \in X$ ") is in $p$.

Proposition 4.2. Suppose that $G$ has $f s g$, witnessed by $p$ and $M_{0}$, and let $X \subseteq G$ be definable. Then the following hold.

(i) $X$ is left generic iff $X$ is right generic (so we just say generic).

(ii) $X$ is generic if and only if every left (right) translate of $X$ meets $M_{0}$.

(iii) $p$ is a generic type, as is any left or right translate of $p$.

(iv) If $X$ is generic and $X=X_{1} \cup X_{2}$ where the $X_{i}$ are definable, then one of $X_{1}, X_{2}$ is generic.

Proof. Before we start, let us note that

$\left(^{*}\right) p^{-1}=\left\{\phi(x): \phi\left(x^{-1}\right) \in p\right\}$ has the property that every right translate of it is finitely satisfiable in $M_{0}$

(i) Suppose $X$ to be left generic. Then for any $c \in G, c X$ is also left generic, so some left translate of $c X$ is contained in $p$ whereby $c X$ is contained in some left translate $g p$ of $p$. By the assumption (on $p, M_{0}$ ), $c X$ meets $M_{0}$, namely there is $b \in G\left(M_{0}\right)$ such that $b \in c X$, so $c^{-1} \in X b^{-1}$. We have shown that every element of $G$ lies in $X b$ for some $b \in G\left(M_{0}\right)$. Compactness implies that finitely many right translates of $X$ cover $G$; namely $X$ is right generic. The other direction (right generic implies left generic) follows from $(*)$ in this proof and symmetry.

(ii) follows from the proof of (i).

(iii) If $X$ is in $p$, then every left translate of $X$ is in a left translate of $p$ and so meets $M_{0}$, whereby $X$ is generic by (ii).

(iv) If $X$ is generic, then $X$ is in a translate of $p$. Thus one of $X_{1}, X_{2}$ is in the same translate of $p$. By (iii) one of $X_{1}, X_{2}$ is generic.

Notice that Proposition 4.2 implies that $G$ has $f s g$, witnessed by $M_{0}$, if and only if every definable generic subset of $G$ meets $M_{0}$ and the complement of every nongeneric set is generic (the latter implies the existence of a generic type, while the first implies that a generic type is finitely satisfiable). 
It follows from (iv) that, assuming that $G$ has $f s g$, the set of nongeneric definable subsets of $G$ forms an ideal $\mathcal{I}$ in the Boolean algebra of all definable subsets of $G$. So for a definable subset $X$ of $G$, the stabilizer of $X$ modulo this ideal, namely $\operatorname{Stab}_{\mathcal{I}}(X)=\{g \in G: g X \Delta X$ is nongeneric $\}$, forms a subgroup of $G$. Note also that $\operatorname{Stab}_{\mathcal{I}}(X)$ is type-definable (by countably many formulas). On the other hand for any global type $q$ of $G, \operatorname{Stab}(q)$ is defined to be the set of $g \in G$ such that $g q=q$. This is clearly a subgroup of $G$, but on the face of it, it has no definability properties.

Corollary 4.3. Suppose that $G$ has $f$ sg. Then the following hold.

(i) There is a bounded number of (global) generic types.

(ii) $G^{00}$ exists.

(iii) For each (global) generic type $p(x), \operatorname{Stab}(p)=G^{00}=\bigcap\left\{\operatorname{Stab}_{\mathcal{I}}(X): X \in p\right\}$.

Proof. (i) Each generic type is finitely satisfiable in $M_{0}$ by Proposition 4.2 (ii). So there are a bounded number of them. (Any global type $p$ which is finitely satisfiable in a model $M_{0}$ is determined by $\left\{X \cap M_{0}: X \in p\right\}$.)

(ii) Let (by part (i)) $\lambda$ be the number of global generic types of $G$. Fix a generic type $p$. Let $H$ be a type-definable subgroup of $G$ of bounded index. So each coset of $H$ is in a translate of $p$. The index of $H$ in $G$ is thus bounded by the number of (left) translates of $p$, which is at most $\lambda$. So we have an absolute bound (independent of the monster model) on the index of type-definable subgroups of $G$ of bounded index, which clearly implies that $G^{00}$ exists.

(iii) Fix a global generic type $p$ of $G$. As $G^{00}$ has bounded index, some translate of $G^{00}$ is in $p$ (namely for some translate $C$ of $G^{00}, p(x)$ implies $x \in C$ ), whereby

(a) $\operatorname{Stab}(p) \subseteq G^{00}$.

On the other hand clearly

(b) $\bigcap\left\{\operatorname{Stab}_{\mathcal{I}}(X): X \in p\right\} \subseteq \operatorname{Stab}(p)$, as $p$ only contains generic definable sets.

So to conclude the proof of (iii), it suffices to prove the following.

(c) For each definable $X \in p, \operatorname{Stab}_{\mathcal{I}}(X) \supseteq G^{00}$.

Suppose $X$ is defined over a small model $M$ containing $M_{0}$. Note that if $g, h \in G$ and $t p(g / M)=t p(h / M)$, then $g X \cap G(M)=h X \cap G(M)$, whereby $g X \Delta h X$ is not satisfiable in $M_{0}$ and hence is nongeneric. It follows that the index of $\operatorname{Stab}_{\mathcal{I}}(X)$ in $G$ is bounded by the number of types over $M$, that is to say, $\operatorname{Stab}_{\mathcal{I}}(X)$ has bounded index in $G$ and hence contains $G^{00}$. This proves (c) and completes the proof of the corollary.

Remark 4.4. If $G$ has $f s g$ and $M_{0}^{\prime}$ is any model, then all generic definable sets meet $G\left(M_{0}^{\prime}\right)$.

Proof. Fix a formula $\phi(x, y)$ and let $k<\omega$. By compactness there is a finite subset $D$ of $G\left(M_{0}\right)$ such that if $X \subseteq G$ is defined by an instance of $\phi(x, y)$ and if $k$ left translates of $X$ cover $G$, then $X$ meets $D$. Let $d$ be a finite tuple enumerating $D$. Then the above property of $d$ can be expressed by a formula without parameters. As this formula is realized in every model $M_{0}^{\prime}$, we are done.

The following will be helpful in carrying out inductive proofs:

Proposition 4.5. Let $G$ be $a \emptyset$-definable group and $N$ a $\emptyset$-definable normal subgroup of $G$. Suppose that $G / N$ and $N$ both have $f s g$. Then so does $G$. 
Proof. Fix a small model $M_{0}$ witnessing that each of $G / N$ and $N$ have $f s g$. (In fact by Remark 4.4, $M_{0}$ can be any submodel of $\bar{M}$.)

We will freely use Proposition 4.2 applied to each of $G / N$ and $N$.

For $X$ a definable subset of $G$, let us define $Y_{X}$ to be $\left\{g / N \in G / N: g^{-1} X \cap N\right.$ is generic in $N\}$. Now $Y_{X}$ is not necessarily definable, so we cannot apply directly Proposition 4.2. But $Y_{X}=\bigcup_{i=1}^{\infty} Y_{X}^{i}$, where $Y_{X}^{i}$ is the set of $g / N \in G / N$ such that $i$ left translates of $g^{-1} X \cap N$ by elements of $N$ cover $N$. Each $Y_{X}^{i}$ is of course definable.

By compactness and the fact that $G / N$ is $f s g$ we have:

Claim 1. Finitely many left translates of $Y_{X}$ cover $G / N$ iff finitely many right translates of $Y_{X}$ cover $G / N$ iff for some $i<\omega, Y_{X}^{i}$ is generic in $G / N$.

We will simply say " $Y_{X}$ is generic in $G / N$ " if the equivalent conditions of Claim 1 hold.

Claim 2. If $Y_{X}$ is generic in $G / N$ and $h \in G$, then each of $Y_{h X}$ and $Y_{X h}$ are generic in $G / N$.

Proof. Just notice that $Y_{X}=(h / N)^{-1} Y_{h X}=Y_{X h}(h / N)^{-1}$.

Claim 3. Suppose $X=X_{1} \cup X_{2}$ where the $X_{i}$ are definable. Then

(i) $Y_{X}=Y_{X_{1}} \cup Y_{X_{2}}$, and

(ii) if $Y_{X}$ is generic in $G / N$, then one of $Y_{X_{1}}, Y_{X_{2}}$ is generic in $G / N$.

Proof. (i) As $N$ has $f s g$, for each $g \in G, g^{-1} X \cap N$ is generic in $N$ if and only if $g^{-1} X_{1} \cap N$ or $g^{-1} X_{2} \cap N$ is generic in $N$.

(ii) Assume that $Y_{X}$ is generic in $G / N$, so there are $h_{1}, \ldots, h_{n} \in G / N$ such that $\bigcup_{j=1, \ldots, n} h_{j} Y_{X}=G / N$. By part (i), $G / N$ is covered by the $h_{j} Y_{X_{1}}$ together with the $h_{j} Y_{X_{2}}$ for $j=1, \ldots, n$. Writing $Y_{X_{1}}$ as $\bigcup_{i<\omega} Y_{X_{1}}^{i}$ and likewise for $Y_{X_{2}}$ and applying compactness, we see (as $G / N$ has $f s g$ ) that either some $h_{j} Y_{X_{1}}^{i}$ is generic in $G / N$ or some $h_{j} Y_{X_{2}}^{i}$ is generic in $G / N$. This suffices.

Claim 4. If $Y_{X}$ is generic in $G / N$, then $X \cap M_{0} \neq \emptyset$.

Proof. By Claim 1, let $i$ be such that $Y_{X}^{i}$ is generic in $G / N$. Hence $Y_{X}^{i} \cap M_{0} \neq \emptyset$. This means precisely that there is $h \in G\left(M_{0}\right)$ such that $h / N \in Y_{X}^{i}$. So $h^{-1} X \cap N$ is generic in $N$ and $h \in G\left(M_{0}\right)$. Now, since $N$ has $f s g$, the set $h^{-1} X \cap N$ contains an element of $G\left(M_{0}\right)$, which clearly implies that $X$ does.

To conclude the proof, for $X$ a definable subset of $G$, let us call $X^{*}$-generic if $Y_{X}$ is generic in $G / N$. By Claims 2 and 3, the family of *-generics is closed under (left or right) translation, and the family of non-*-generics forms a proper ideal. Hence there is a global *-generic type $p$ of $G$, and moreover by Claim 4 , every translate of $p$ is finitely satisfiable in $M_{0}$. This shows that $G$ has $f s g$.

Remark 4.6. The $f s g$ property can also be formulated in terms of measures. Namely, we say that a group $G$ has $f s g_{m}$ if there is a Keisler measure $\mu$ on $G$ and there is some small model $M_{0}$ of $\bar{M}$ such that for every $g \in G$, the measure $g \mu$ (defined as $g \mu(X)=\mu(g X))$ is finitely satisfiable in $M_{0}$. It turns out that these formulations are equivalent:

If $G$ has $f s g$, then the generic type gives the desired 0-1 measure. For the converse, one goes through the proof of Proposition 4.2, using the $f s g_{m}$ assumption instead of $f s g$. 
One could ask if the results of this section hold under the weaker assumption that there exists a type over a large saturated model, with a small number of left translates. However variants of the following example will show that various lemmas can fail, even for rank one simple theories with a translation invariant type.

Example 4.7. Let $T_{0}$ be the theory of vector spaces over $G F(2)$ with a symmetric irreflexive binary relation $\mathrm{R}$ (bearing no particular relation to + ). Let $T$ be the model completion of $T_{0}, M \models T$. Let $C \subseteq M$ and $D \subseteq M \backslash\{0\}$ be arbitrary subsets. Then $\{R(x+b, c): c \in C\} \cup\{\neg R(x+b, c): c \notin C\} \cup\{R(x+c, x+b)$ : $b-c \in D\} \cup\{\neg R(x+c, x+b): b-c \notin D\}$ determines a complete type, which is $M$-translation invariant. But there are essentially no generic formulas.

\section{Definably amenable Groups}

It is a convenient time to introduce the notion of "definable amenability". Recall that an abstract (or discrete) group $G$ is said to be amenable if there exists a left invariant finitely additive probability measure on the family of all subsets of $G$. Any solvable group is amenable.

Definition 5.1. Let $G$ be a definable group. We call $G$ definably amenable if there is a left invariant Keisler measure on $G$.

Remark 5.2. (i) Any amenable group is definably amenable.

(ii) Suppose $T$ has a model $M_{0}$ such that $G$ is defined over $M_{0}$ and $G\left(M_{0}\right)$ has a compact (Hausdorff) group topology such that every definable subset of $G$ is Haar measurable. Then $G$ is definably amenable.

(iii) If $K$ is a (saturated) algebraically closed-valued field and $n>1$, then $S L(n, K)$ is not definably amenable.

(iv) If $R$ is an expansion of a real closed field, then $P S L(2, R)$ is not definably amenable.

(v) $S O(3, \mathbb{R})$ is definably amenable, but not amenable as a pure group.

Proof. (ii) is proved by Construction (*) applied to the (unique) normalized Haar measure on $G\left(M_{0}\right)$.

(iii) This follows by a similar proof to that in [12] showing that $S L(n, K)$ has no definable left generic type.

(iv) Suppose $\mu_{1}$ is a left invariant Keisler measure on $P S L(2, R)$. Recall the transitive action of $S L(2, R)$ on $\mathbb{P}^{1}(R)=R \cup\{\infty\}$. Define a Keisler measure $\mu$ on $\mathbb{P}^{1}(R)$ by $\mu(X)=\mu_{1}(\{g \in P S L(2, R): g \cdot 0 \in X\})$. Then $\mu(h X)=\mu_{1}(\{g \in$ $\left.\left.P S L(2, R): h^{-1} g \cdot 0 \in X\right\}\right)=\mu(X)$. But let $U$ be a small ball around 0 ; then using inversion, we find $g U$, a ball around $\infty$; while using multiplication, we can find $h U$ such that $\mathbb{P}^{1}(R)=g U \cup h U$. So $\mu(U) \geq 1 / 2 \mu\left(\mathbb{P}^{1}(R)\right)$. This is true for an arbitrary small ball around any point, e.g. $0,1, \infty$, giving $3 / 2 \leq 1$, a contradiction.

(v) Every definable set is Lebesgue measurable. The pure group statement is due to Hausdorff, Banach and Tarski; see below.

Before continuing, we take the opportunity to give a characterization of definable amenability (and the construction of an invariant Keisler measure on $G$ from a suitable Grothendieck group of $G$ ). Fix a definable group $G$. By a nonnegative cycle in $G$ we mean a "finite disjoint union" of definable subsets of $G$. Notationally, consider a nonnegative cycle as $\left\{k_{1} X_{1}, \ldots, k_{n} X_{n}\right\}$ where the $k_{i}$ are nonnegative integers and $X_{i}$ are pairwise distinct definable subsets of $G$. There is the obvious 
notion of a map between two such cycles being definable, 1-1 and given by piecewise left translations.

Definition 5.3. A definable paradoxical decomposition of $G$ is a definable 1-1 piecewise translation from the disjoint union of $G$ and $Y$ to $Y$, for some nonnegative cycle $Y$.

This is a variant of a notion due to Hausdorff. He actually considered a stronger notion that would rule out the existence of any invariant finitely additive measure, not necessarily nonnegative. His construction of a paradoxical decomposition of the two-dimensional sphere, or of $S O(3, \mathbb{R})$, completed by Banach and Tarski, requires the axiom of choice and is not represented in a definable way. On the other hand we have

Proposition 5.4. $G$ is definably amenable if and only if $G$ does not admit a definable paradoxical decomposition.

Before starting the proof, we introduce the relevant Grothendieck (semi)group. Let $K_{\text {semi }}(G)$ be the semigroup whose elements are the nonnegative cycles $\sum_{i} k_{i} X_{i}$ in $G$ modulo the equivalence relation of being in definable bijection by piecewise left translations. A typical element of $K_{\text {semi }}(G)$ can be written in the form $k_{i}[X]_{\text {semi }}$ where $[X]_{s e m i}$ is the class of the definable set $X$ in $K_{s e m i}(G)$. Addition in the semigroup is the obvious thing.

Let us make a further identification: let $x_{1}, x_{2} \in K_{\text {semi }}(G)$. Define $x_{1} \sim_{0} x_{2}$ if there is $y \in K_{\text {semi }}(G)$ such that $x_{1}+y=x_{2}+y$. Then the collection of $\sim_{0}$-classes, together with formal inverses, constitutes the Grothendieck group $K_{0}(G)$. The class in $K_{0}(G)$ of a definable subset $X$ of $G$ is denoted $[X]_{0}$. (Likewise for a nonnegative cycle $Y$.)

Proof of Proposition 5.4. Suppose first that $\mu$ is a left invariant Keisler measure on $G$. Then a definable paradoxical decomposition could not exist since then we would have $\mu(G)+\mu(Y) \leq \mu(Y)$, contradicting $\mu(G)=1$.

Conversely suppose $G$ has no definable paradoxical decomposition. Let $P_{0}$ be the subsemigroup of $K_{0}(G)$ generated by the sets $[X]_{0}$ where $X$ is definable.

Claim. $-n[G]_{0} \notin P_{0}$ for all $n>0$.

Proof. Otherwise $-n[G]_{0}=[Y]_{0}\left(\right.$ in $\left.K_{0}(G)\right)$ for some nonnegative cycle $Y$. But then $n[G]_{0}+[Y]_{0}=0$ in $K_{0}(G)$, so $n[G]_{\text {semi }}+Y_{\text {semi }}+[Z]_{\text {semi }}=[Z]_{\text {semi }}$ in $K_{\text {semi }}(G)$ for some nonnegative cycle $Z$. But then clearly there is a definable injective piecewise translation map from the disjoint union of $G$ and $Z$ into $Z$, contradicting our assumption.

Let $B$ be the tensor product of $\mathbb{Q}$ with $K_{0}(G)$, and let

$$
P=\left\{\alpha x: \alpha \in \mathbb{Q}, \alpha>0, x \in P_{0}\right\} .
$$

By the claim $-[G]_{0} \notin P$. Let $P^{\prime}$ be a maximal subset of $B$ containing $P$, closed under multiplication by positive rationals and addition and such that $-[G]_{0} \notin P^{\prime}$. Define a partial ordering on $B: x \leq y \Longleftrightarrow y-x \in P^{\prime}$.

Claim. $\leq$ is a total ordering on $B$.

Proof. We have to show that for any $a \in B$, either $a \in P^{\prime}$ or $-a \in P^{\prime}$. If $a \notin P^{\prime}$, let $P^{\prime \prime}=\left\{x+\alpha a: x \in P^{\prime}, \alpha \in \mathbb{Q}, \alpha>0\right\}$. Then by maximality $-[G]_{0} \in P^{\prime \prime}$, i.e. $-[G]_{0}=x+\alpha a, x \in P$, so $-a=\alpha^{-1}\left([G]_{0}+x\right) \in P^{\prime}$. 
Now exists a unique order-preserving semigroup homomorphism $h: B \rightarrow \mathbb{R} \geq 0$ such that $[G]_{0}$ goes to 1 . (Namely $h(b)=\alpha$ iff $\left[b:[G]_{0}\right]=[\alpha: 1]$ in the sense of $[9$, V. Def. 5, i.e. for all $m, n \in \mathbb{N}, m b<n\left[G_{0}\right]$ iff $\left.m \alpha<n \cdot 1\right)$. Let $\mu(X)=h\left([X]_{0}\right)$; this is clearly a left invariant Keisler measure on $G$.

Definable amenability of volumes. We will see in Section 8 that definably compact groups in o-minimal structures are definably amenable. The proof uses a deep structure theory for such groups. In the following paragraphs, not otherwise used in this paper, we consider a similar amenability property of definable compact definable sets. We do not know if this property holds in all $o$-minimal theories, but when it does, we give a soft proof of definable amenability of definable compact groups. In particular this is valid for $o$-minimal expansions of RCF that are finitely satisfiable in expansions of $(\mathbb{R},+, \cdot)$.

The proof actually yields more: that any definable group $G$, not necessarily definably compact, is definably amenable for compact sets. By definition this means: let $D e f_{b d d}(G)$ be the family of definable subsets of definably compact subsets of $G$. Then for any $X \in D e f_{b d d}(G)$ with nonempty interior, there exists a translation invariant finitely additive $\mu: D e f_{b d d}(G) \rightarrow \mathbb{R}^{\geq 0} \cup\{\infty\}$ with $\mu(X)=1$.

Let $T$ be an $o$-minimal expansion of RCF, and fix $n \geq 1$. By "almost all" we will mean: away from a definable set of dimension $<n$. If $f: R^{n} \rightarrow R^{n}$ is definable, $|J f|(c)$ denotes the absolute value of the determinant of the matrix of partial derivatives of $\phi$ at $c$; it exists almost everywhere. Let $V[n]$ be the set of bounded definable functions $R^{n} \rightarrow R^{\geq 0}$ with bounded support. By an isomorphism $\phi: f \rightarrow g$ we mean a definable bijection $\phi$ from a definable set containing the support of $f$ to one containing the support of $g$, such that $f(x)=|J \phi|(x) \cdot g(\phi(x))$ almost everywhere. More generally, define $f \sim g$ if one can write $f=\sum_{i=1}^{n} f_{i}, g=\sum_{i=1}^{n} g_{i}$ with $f_{i}, g_{i}$ isomorphic. Let $[f]$ denote the $\sim$-class of $f$, and let $K_{\text {semi }}(V[n])=\{[f]: f \in V[n]\}$. Define $[f]+[g]=[f+g]$. Let $K(V[n])$ be the corresponding group. We say that $T$ is definably amenable for volumes if for each $n$ and any $f \in V[n]$, either $f=0$ a.e. or there exists an order-preserving semigroup homomorphism $I: K_{\text {semi }}(V[n]) \rightarrow \mathbb{R} \geq 0 \cup\{\infty\}$ with $0<I(f)<\infty$.

Proposition 5.5. Conditions (1) and (2) are equivalent. (3) implies (1). Each of (4), (5) implies (3). (1) implies (6) and (6) implies (7).

(1) $T$ is definably amenable for volumes.

(2) If $f \in V[n]$ and for some $m$ and $g \in V[n],(m+1) f+g \sim m f$, then $f=0$ almost everywhere.

(3) If $f, h \in V[n]$ and $f \sim f+h$, then $h=0$ a.e.

(4) Every finite $T_{0} \subseteq T$ has a complete archimedean model.

(5) $T$ has definable primitives: for every definable function $f: R \rightarrow R$ in a model of $T$, there exists a definable function $F$ such that almost everywhere $F^{\prime}=f$.

(6) Every definable group $G$ of $T$ is definably amenable for compact sets.

(7) Every definably compact group in $T$ is definably amenable.

Proof. The proof of the equivalence of (1) and (2) and the implication (3) $\rightarrow(2)$ is similar to the proof of Proposition 5.4 
If (3) fails, then there exists a finite $T_{0}$ describing the situation. For instance if there exists an isomorphism $\phi: f \rightarrow f+h$, then $\phi$ is differentiable away from a set $Y$ of dimension $n-1$; there exist $W_{1}, \ldots, W_{k} \subseteq R^{n-1}$ and continuously differentiable $e_{i}: W_{i} \rightarrow Y$ with $\bigcup_{i=1}^{k} e_{i}\left(W_{i}\right)=Y$; and $f+h=|J(\phi)| \cdot f \circ \phi$ away from $Y$. $T_{0}$ can state this, as well as the boundedness and piecewise differentiability of $f, h$, and the fact that $h>0$ on some open set. If $T_{0}$ has a complete archimedean model $N=$ $\left(\mathbb{R},+, \cdot, f^{N}, h^{N}, \ldots\right)$, then $f^{N}, h^{N}$ are bounded integrable functions and $\phi^{N}$ is $C^{1}$ away from $Y^{N}$, so by the change of variable formula $\int f^{N}=\int(f+h)^{N}$ (where $\int$ is the Lebesgue or Riemann integral). But also $\int h^{N}>0$, a contradiction. This shows that (4) implies (3). The proof that (5) implies (3) is similar: by a compactness argument, a function $f\left(x_{1}, \ldots, x_{n}\right)$ has a definable primitive $F_{1}\left(x_{1}, \ldots, x_{n}\right)$ with respect to the first variable, i.e. $\partial F_{1} / \partial x_{1}=f$ a.e. Now one can define integration using iterated integrals and prove the change of variable formula and additivity using o-minimality. The proof of (4) implies (3) used no more than this.

(7) is obviously a special case of $(6)$.

To prove (6) from (1), let $n=\operatorname{dim}(G)$. Fix an identification of some neighborhood of 1 in $G$ with an open neighborhood of 0 in $R^{n}$. Let $K_{0}$ be the set of subsets $Y$ of $G$ contained in $b(\operatorname{int}(U))$ for some injective continuously differentiable definable map $b: U \rightarrow G, U$ a definably compact subset of $R^{n}$ with interior int $(U)$. We begin by defining a map $\psi: K_{0} \rightarrow K_{\text {semi }}(V[n])$.

For $g \in G$, let $T_{g}: G \rightarrow G, T_{g}(x)=g^{-1} x$. Given $Y \in K_{0}$, find a definably compact set $U \subseteq R^{n}$ and a definable injective $C^{1}$ map $b: U \rightarrow G$, with $Y \subseteq$ $b(\operatorname{int}(U))$. Let $f(x)=0$ for $x \notin b^{-1}(Y)$, and for $x \in b^{-1}(Y)$ let $f(x)=|J g|(x)$, where $g(y)=T_{b(x)} \circ b$. (Here we use the identification of a neighborhood of 1 with a neighborhood of 0 in $\mathbb{R}^{n}$; so $g: U \rightarrow \mathbb{R}^{n}$, and the Jacobian $J g$ is defined.) By continuity and definable compactness, $f$ is bounded on $U$. If we pick a different $b^{\prime}: U^{\prime} \rightarrow Y$, with corresponding $g^{\prime}, f^{\prime}$, then $b^{\prime}=b \circ e$ for some $e: U^{\prime} \rightarrow U$ (defined on a neighborhood of the support of $b$ ), namely $e\left(u^{\prime}\right)=b^{-1}\left(b^{\prime}\left(u^{\prime}\right)\right)$ on $\left(b^{\prime}\right)^{-1}(b(\operatorname{int}(U)))$. We have $g^{\prime}=g \circ e,\left|J g^{\prime}\right|(x)=|J g|(e(x))|J e|(x)$ so that $f$ is isomorphic to $f$ and $[f]=\left[f^{\prime}\right] \in K_{\text {semi }}(V[n])$. Hence $[f]$ does not depend on the choice of $(U, b)$ and we can define $\psi(Y)=[f]$.

Given $h \in G$, let $b^{\prime \prime}=h b$; then $T_{b^{\prime \prime}(x)} \circ b^{\prime \prime}=T_{b(x)} \circ b$, so $\psi(h U)=\psi(U)$. Thus $\psi$ induces a well-defined map $K_{1} \rightarrow K_{\text {semi }}(V[n])$, where $K_{1}=\left\{[Y]: Y \in K_{0}\right\} \subseteq$ $K_{\text {semi }}(G)$. It is clear that $\psi(a+b)=\psi(a)+\psi(b)$ when $a, b, a+b \in K_{1}$ and that $a+b \in K_{1}$ implies $a \in K_{1}$. It follows that $\psi$ extends to homomorphism of ordered semigroups $\sum K_{1} \rightarrow K_{\text {semi }}(V[n])$, where $\sum K_{1}$ is the semigroup generated by $K_{1}$.

According to [3], for any definably compact $Z \subseteq G$ there is a $C^{1}$ group manifold structure on $G$ with finite chart $\left\{b_{i}: W_{i} \rightarrow G: i=1, \ldots, r\right\}$ (with the $W_{i}$ open subsets of $R^{n}$ ) and closed bounded $U_{i} \subseteq W_{i}$, such that $X \subseteq \bigcup_{i=1}^{r} b_{i}\left(\operatorname{int}\left(U_{i}\right)\right)$. Hence $\sum K_{1}=K_{\text {semi }}(G)$.

If $X$ has nonempty interior, then $\psi(X)$ cannot vanish almost everywhere, so by (1) there exists a homomorphism $\mu: K_{\text {semi }}(V[n]) \rightarrow \mathbb{R}^{\geq 0} \cup\{\infty\}$ with $\mu(\psi(X))=$ $a>0$. Now $(1 / a) \mu \circ \psi$ demonstrates (6).

It is of course possible to combine (4) and (5), i.e. it suffices that every finite $T_{0} \subseteq T$ be extendible to an $o$-minimal theory with definable primitives, or to one with an archimedean model.

Question 5.6. Is every o-minimal theory amenable for volumes? 


\section{Groups with NIP}

Here we concentrate on definable groups in theories with NIP.

Suppose that $\mu$ is a Keisler measure on a definable group $G$. Then for any $g \in G$, we have another Keisler measure $g \mu$ on $G$, namely $g \mu(X)=\mu(g X)$. We say that $\mu$ is left invariant if $g \mu=\mu$ for all $g \in G$. Likewise for right invariant. The existence of a left invariant type of $G$ is a very strong property. For example if $G$ is stable, this implies that $G$ is connected and the left invariant type is the unique generic type of $G$. However, even if there is NO invariant type, one may hope for there to exist an invariant measure.

The next proposition, due to Shelah [27, gives the existence of $G^{00}$ for any definable (or even type-definable) group $G$ in a theory with NIP. We had originally proved this under the additional assumption that $G$ was definably amenable. In any case thanks to Shelah for allowing us to include the result and a proof.

Proposition 6.1. Assume $T$ has NIP. Let $G$ be a definable group in $\bar{M}$, defined over $\emptyset$ say. Then $G$ has a smallest type-definable subgroup of bounded index. If $G^{00}$ is such, then it is type-definable over $\emptyset$ and has index at most $2^{|T|}$.

Proof. It is easy to see that any type-definable subgroup of $G$ is the intersection of a family of subgroups each of which is type-defined by countably many formulas (see for example Remark 1.4(ii) in [4]). So it suffices to prove that any subgroup $H$ of $G$ which is type-defined by countably many formulas and has bounded index in $G$ has only a bounded number of distinct conjugates (under automorphisms of the ambient structure). So let us suppose, for a contradiction, that $a$ is a countable tuple, $H_{a}$ is type-definable by a countable partial type $\Sigma(x, a)$ over $a, H_{a}$ is a subgroup of bounded index in $G$, and that $\left\{H_{a^{\prime}}: t p\left(a^{\prime}\right)=t p(a)\right\}$ is unbounded (where $H_{a^{\prime}}$ is type-defined by $\left.\Sigma\left(x, a^{\prime}\right)\right)$. So by Erdös-Rado we have some indiscernible sequence $\left\langle a_{i}: i<\omega\right\rangle$ of realizations of $p=t p(a)$ such that $H_{a_{i}} \neq H_{a_{j}}$ for $i \neq j$.

Claim 1. Fix $i_{0}<\omega$. Then $\bigcap\left\{H_{a_{j}}: j<\omega, j \neq i_{0}\right\}$ is NOT contained in $H_{a_{i_{0}}}$.

Proof of Claim 1. Suppose otherwise. We can "stretch" the indiscernible sequence $\left\langle a_{i}: i<\omega\right\rangle$ by inserting some $\left(b_{\alpha}: \alpha<\kappa\right)$ in place of $a_{i_{0}}$ (for any $\kappa$ ). But then each $H_{b_{\alpha}}$ contains $\bigcap_{j \neq i_{0}} H_{j}$. But $\alpha \neq \beta$ implies $H_{b_{\alpha}} \neq H_{b_{\beta}}$. So for any $\kappa$ we can find at least $\kappa$ many distinct subgroups of $G$ each of which contain $\bigcap_{j \neq i_{0}} H_{j}$. As the latter has bounded index in $G$, we get a contradiction, proving the claim.

The claim clearly applies also to any stretching $\left\langle a_{\alpha}\right\rangle$ of the indiscernible sequence $\left\langle a_{i}: i<\omega\right\rangle$. So for each $\alpha$, let $c_{\alpha}$ be such that $c_{\alpha} \notin H_{\alpha}$ but $c_{\alpha} \in H_{\beta}$ for all $\beta \neq \alpha$. Again by Erdös-Rado we may assume that the sequence $\left\langle\left(a_{\alpha}, c_{\alpha}\right): \alpha<\kappa\right\rangle$ is indiscernible.

We may assume $\Sigma(x, a)=\left\{\phi_{n}(x, a): n<\omega\right\}$ where $n<m$ implies $\models \phi_{m}(x, a) \rightarrow$ $\phi_{n}(x, a)$.

Claim 2. There is $n<\omega$ such that for any $\alpha$ and any $d_{1}, d_{2} \in H_{\alpha}, \models \neg \phi_{n}\left(d_{1} \cdot c_{\alpha}\right.$. $\left.d_{2}, a_{\alpha}\right)$.

Proof of Claim 2. As $t p\left(a_{\alpha}, c_{\alpha}\right)$ does not depend on $\alpha$, it is enough to prove it for a fixed $\alpha$. As $c_{\alpha} \notin H_{\alpha}$, we have the implication:

$y_{1}, y_{2} \in H_{\alpha} \models \vee_{n} \neg \phi_{n}\left(y_{1} \cdot c_{\alpha} \cdot y_{2}, a_{\alpha}\right)$. Now apply compactness.

We may clearly assume $n=0$ in Claim 2 . 
Claim 3. For each finite $w \subset \kappa$ there is $d_{w}$ such that for all $\alpha, \models \phi_{0}\left(d_{w}, a_{\alpha}\right)$ iff $\alpha \notin w$.

Proof of Claim 3. Let $d_{w}$ be the product of the $c_{\beta}$ for $\beta \in w$. So if $\alpha \notin w$, then as $c_{\beta} \in H_{\alpha}$ for each $\beta \in w, d_{w} \in H_{\alpha}$ and hence satisfies $\phi_{0}\left(x, a_{\alpha}\right)$. On the other hand if $\alpha \in w$, then we can write $d_{w}$ as $d_{1} \cdot c_{\alpha} \cdot d_{2}$ where $d_{1}, d_{2} \in H_{\alpha}$ (by an argument as above). So then we apply Claim 2.

Claim 3 shows that $T$ has the independence property, a contradiction. So $G^{00}$ exists. Its type-definability over $\emptyset$ follows by uniqueness (any type-definable set which is $\emptyset$-invariant is type-definable over $\emptyset$, by quantifying out the parameters and using saturation). The bound on the index is clear too.

The existence of $G^{00}$ (for $G$ a definable group in a saturated model of $T$ ) had been proved earlier in various special cases, for example for o-minimal theories in 4. In fact the latter proved in addition that $G / G^{00}$ is a compact Lie group. For groups definable over $\mathbb{Q}_{p}$ in a model of $T h\left(\left(\mathbb{Q}_{p}\right)_{a n}\right)$ this was done in [16]. For groups definable in Pressburger Arithmetics, it follows from work of Onshuus 15.

Here is an application of Proposition 6.1. Let us fix a compact Hausdorff group $\langle G, \cdot, \ldots\rangle$ equipped with additional first order structure. We use the term $G$ to also denote this structure. Let us assume that (i) $T h(G)$ has the NIP, (ii) any definable subset of $G$ is Haar measurable (with respect to the unique normalized Haar measure on $G$ ), and (iii) there is a neighbourhood basis of the identity of $G$ consisting of definable sets, say $U_{i}$ for $i \in I$.

Let $G^{*}$ be a saturated elementary extension of $G$. So $\bigcap_{i \in I} U_{i}^{*}$ is the group of "infinitesimals", denoted by inf $\left(G^{*}\right)$ of $G^{*}$, and the quotient group (with the logic topology) is precisely $G$. By Proposition 6.1, $\left(G^{*}\right)^{00}$ exists, and in fact we have

Claim. $\left(G^{*}\right)^{00}$ is precisely the group $\inf \left(G^{*}\right)$ of infinitesimals of $G^{* *}$.

Proof. By Proposition 6.1 $\left(G^{*}\right)^{00}$ is type-definable over $\emptyset$. As we already know that $\inf \left(G^{*}\right)$ is type-definable and of bounded index, it suffices to prove that any subgroup $H$ of $G^{*}$ which is type-definable over $G$ by a countable set of formulas, and has bounded index, contains $\inf \left(G^{*}\right)$. Let $H$ be such, and suppose $H=\bigcap_{n} X_{n}$, where $X_{n}$ is definable over $G$. We may assume that $X_{n}^{-1} \cdot X_{n} \subseteq X_{n-1}$ for all $n>0$. Fix $n$. As $H$ has bounded index in $G^{*}$, finitely many translates of $X_{n}(G)$ cover $G$ whereby the Haar measure of $X_{n}(G)$ is $>0$.

It follows (cf. the chapter on convolutions in [11]) that $\left(X_{n}^{-1} \cdot X_{n}\right)(G)$ has interior in $G$, so $\left(X_{n-1}^{-1} \cdot X_{n-1}\right)(G)$ contains an open neighbourhood of the identity of $G$. Thus $X_{n-2}(G)$ contains some $U_{j}$. Hence $H$ contains inf $\left(G^{*}\right)$, and the claim is proved.

Now measures come back into the picture. The following was proved in the stable case in [14].

Proposition 6.2. Suppose $T$ has NIP and $G$ is a $\emptyset$-definable group in $\bar{M}$ with $f s g$. Then there is a left invariant Keisler measure $\mu$ on $G$, which is moreover finitely satisfiable in some small model $M_{0}$.

Proof. We will use Proposition 4.2 and Corollary 4.3. Let us fix a global generic type $p$ of $G$ over $\bar{M}$, such that $p(x)$ implies $x \in G^{00}$. The measure we construct will depend on $p$. We will first prove the proposition in the case where $T$ is countable. By Remark 4.4 let us fix a countable model $M_{0}$ such that all generic definable 
subsets of $G$ meet $G\left(M_{0}\right)$. Let $\mathbf{m}$ be the (unique) normalized Haar measure on the compact group $G / G^{00}$.

Claim. Let $X \subseteq G$ be definable. Then the following hold. $G^{00}$.

(i) For $g \in G$, whether or not $g X \in p$ depends only on the coset of $g$ modulo

(ii) $\left\{g / G^{00}: g X \in p\right\}$ is a Borel subset of $G / G^{00}$ (so is Haar measurable).

Proof. (i) follows because $\operatorname{Stab}(p)=G^{00}$.

(ii) By Remark 4.4 let $M_{0}$ be a countable model such that $X$ is over $M_{0}$ and all generic definable subsets of $G$ meet $M_{0}$. In particular $p$ is finitely satisfiable in $M_{0}$. By Corollary 3.9, there are partial types $\Psi_{i}(y)$ over $M_{0}$ for $i<\omega$ such that for $g \in G, g X \in p$ iff $\models \bigvee_{i<\omega} \Psi_{i}(g)$. Let $C_{i}$ be the closed subset of $G / G^{00}$ determined by $\Psi_{i}(y)$, namely the image of the solution set of $\Psi_{i}$ under the natural map taking $G$ to $G / G^{00}$. Then by part (i) of the claim, $\left\{g / G^{00}: g X \in p\right\}$ is precisely $\bigcup_{i} C_{i}$, hence Borel.

By the claim, we can define $\mu_{p}(X)=\mathbf{m}\left(\left\{g / G^{00}: g X \in p\right\}\right)$. Then $\mu_{p}$ is finitely additive. For left invariance, let $g^{\prime} \in G$. Then $\left\{g / G^{00}: g \in g^{\prime} X\right\}=\left\{g / G^{00}: g \in\right.$ $X\} g^{\prime} / G^{00}$, so by right invariance of $\mathbf{m}, \mu_{p}\left(g^{\prime} h X\right)=\mu_{p}(X)$.

Finally let us note that $\mu_{p}(X)>0$ if and only if $X$ is generic. Right implies left is true by invariance of $\mu_{p}$. But if $X$ is nongeneric, then no translate of $X$ is in $p$, so $\left\{g / G^{00}: g X \in p\right\}$ is empty, and hence $\mu_{p}(X)=0$.

As we already know that every generic definable subset of $G$ meets $G\left(M_{0}\right)$ for some small model $M_{0}$, we obtain finite satisfiability of $\mu_{p}$ in $M_{0}$.

So we have proved the proposition when $T$ is countable. For the general case, given a definable subset $X$ of $G$, let $L_{0}$ be a countable sublanguage of $L$ in which $G$ and $X$ are definable. Let $p_{0}$ be the reduct of $p$ to $L_{0}$ and let $G_{0}^{00}$ be the smallest $L_{0^{-}}$ type-definable subgroup of $G$ of bounded index. Let $f$ be the canonical surjective homomorphism from $G / G^{00}$ to $G / G_{0}^{00}$. Clearly $f$ is continuous. Let $U=\left\{g / G^{00}\right.$ : $g X \in p\}$ and $U_{0}=\left\{g / G_{0}^{00}: g X \in p_{0}\right\}$. Then $U=f^{-1}\left(U_{0}\right)$. But by the claim in the countable case, $U_{0}$ is Borel. Hence $U$ is also Borel. So the claim holds in general, and as above we obtain our measure $\mu_{p}$.

It is natural to ask whether the measure $\mu_{p}$ defined above indeed depends on the type $p$ or not. This and related issues will be tackled in a subsequent paper.

Our final result of this section will provide in a sense the missing link in the proof of the $o$-minimal conjectures.

Proposition 6.3. Suppose that $T$ has NIP and $G$ is a group definable in $\bar{M}$ such that $G$ is definably amenable and the set $\mathcal{I}$ of non-(left) generic definable subset of $G$ forms an ideal. Then

(i) there are only a bounded number of definable subsets of $G$ modulo the equivalence relation $X \sim \sim_{\mathcal{I}} Y(X \Delta Y \in \mathcal{I})$,

(ii) for each definable left generic $X \subseteq G, \operatorname{Stab}_{\mathcal{I}}(X)(=\{g \in G: g X \Delta X$ is nongeneric $\}$ ) is a (type-definable) subgroup of bounded index.

Proof. Let $\mu$ be a left invariant Keisler measure on $G$. Note that if $X$ is a left generic definable subset of $G$, then $\mu(X)>0$ (as finitely many left translates of $X$ cover $G$ and these have all the same $\mu$-measure as $X)$. So if there are unboundedly 
many $\sim_{\mathcal{I}}$-classes, there will also be unboundedly many $\sim_{\mu}$-classes, contradicting Corollary 3.4. This proves (i).

(ii) follows immediately.

\section{INTERLUdE: IND-DEFINABLE AND LOCALLY COMPACT GROUPS}

As one of the authors remarked, "It seems a pity to lose $S L_{2}(\mathbb{R})$." So we give the notion of an Ind-definable group, point out that quotienting by a type-definable normal subgroup of bounded index yields a locally compact group, and develop analogues of some of the results so far for Ind-definable groups. We also state an appropriate version of the $o$-minimal conjectures from [23]. In any case we will be brief.

We still work in a saturated model $\bar{M}$. Ind-definable stands for "inductive limit of definable sets". For notational reasons we will take the index set to be $\mathbb{N}$. So an Ind-definable set $X$ will be by definition a sequence $\left(X_{n}: n \in \mathbb{N}\right)$ of definable sets together with definable embeddings $f_{n}: X_{n} \rightarrow X_{n+1}$ for $n \in N$. The points of $X$ correspond to sequences $\left(x, f_{n_{0}}(x), f_{n_{0}+1}\left(f_{n_{0}}(x)\right), \ldots\right)$ for some $x \in X_{n_{0}}$ and $n_{0} \in \mathbb{N}$. It is convenient to view the $f_{n}$ as inclusion maps, and so $X$ as the increasing union $\bigcup_{n} X_{n}$. There are natural notions of an Ind-definable relation on $X$ and Ind-definable functions between Ind-definable sets. For example an Inddefinable function $g$ between $X=\bigcup_{n} X_{n}$ and $Y=\bigcup_{n} Y_{n}$ is a function from $X$ to $Y$ such that for every $m, n,\left\{x \in X_{m}: g(x) \in Y_{n}\right\}$ is definable and the restriction of $g$ to this set is definable. We also have the obvious notion of an Ind-definable set, function, ... being defined over a given set $A$ of parameters.

Definition 7.1. An Ind-definable group $G$ is something of the form $G=\bigcup_{n} G_{n}$ where $G_{n}$ are definable sets, $m: G \times G \rightarrow G$ is a group operation and when restricted to $G_{n} \times G_{n}$ has values in $G_{n+1}$ (and is definable), and inversion when restricted to $G_{n}$ has values also in $G_{n}$.

We could also say that an Ind-definable group $G$ is a group object in the category of Ind-definable sets, noting that up to isomorphism $G$ has the explicit form given in Definition 7.1.

By a definable subset of $G$ we mean a definable subset of some $G_{n}$. Likewise a complete type extending $G$ will be "concentrated" on some $G_{n}$.

For various reasons we will assume that

$(* *) G_{0}$ generates $G$ as a group.

Examples. A basic example we have in mind for an Ind-definable group is a subgroup of a definable group $G$ that is generated by a definable set $G_{0} \subseteq G$ (such groups were called "V-definable groups" in [20] and "locally definable" in 6]). Another is the universal cover of $\langle[0,1),+(\bmod 1)\rangle$, obtained as an increasing union of intervals $[-n, n]$ and the obvious group operation. The group of definable automorphisms of a definable group $G$, say in a countable language, can also be viewed as an Ind-definable group, where the $G_{n}$ 's in the definition are obtained via the various definable families of automorphisms of $G$. Finally, "an infinite dimensional" example is, for a definable group $G$, the increasing union of $G, G \times$ $G, \ldots, G^{n}, \ldots$, with the group operation acting coordinatewise (such spaces are called by A. Piekosz, in preliminary notes, "weakly definable spaces"). 
Here are some analogues of the basic notions:

Definition 7.2. Let $G=\bigcup_{n} G_{n}$ be an Ind-definable group.

(i) Let $X$ be a definable subset of $G$ (i.e. of some $G_{n}$ ). We call $X$ left generic in $G$ if for each $m$ finitely many left translates of $X$ by elements of $G$ cover $G_{m}$.

(ii) By a type-definable subgroup of $G$ we mean a subgroup $H$ of $G$ which is at the same time a type-definable subset of some $G_{n}$.

(iii) By a Keisler measure on $G$ (or on any Ind-definable set for that matter) we mean a finitely additive real-valued function $\mu$ on definable subsets of $G$; namely for every definable subset $X$ of $G, \mu(X) \geq 0, \mu(\emptyset)=0$ and if $X, Y$ are disjoint definable subsets of $G$, then $\mu(X \cup Y)=\mu(X)+\mu(Y)$. (But note that we do not require there be a finite bound on the measures of definable sets).

Note a difference with the usual situation: If $G$ is Ind-definable, it may have NO type-definable subgroup of bounded index (because $G$ itself is not type-definable). In any case if $G$ has a smallest type-definable subgroup of bounded index, we will call it $G^{00}$ and say " $G^{00}$ exists".

As in Section 4, we will say that the Ind-definable (over $\emptyset$ ) group $G=\bigcup_{n} G_{n}$ has finitely satisfiable generics if there is a global complete type $p(x)$ of $G$ (namely $p(x) \rightarrow$ " $x \in G_{n}$ " for some $n$ ) such that every left translate of $p$ by an element of $G$ is finitely satisfiable in some fixed small model $M_{0}$.

The material from Section 4 generalizes as follows:

Proposition 7.3. Suppose the Ind-definable group $G$ has $f s g$. Then the following hold.

(i) Any definable subset $X$ of $G$ is left generic iff right generic iff every left (right) translate meets $M_{0}$.

(ii) There is a complete global generic type of $G$ (in fact $p$ as in the definition of $f s g$ will be such, as well as any translate of $p)$.

(iii) If $X$ is a definable subset of $G$ which is generic in $G$ and $X=X_{1} \cup X_{2}$ with $X_{i}$ definable, then $X_{1}$ or $X_{2}$ is generic in $G$.

(iv) There is a smallest subgroup of $G$ which has bounded index and is invariant over some small set of parameters.

(v) $G^{00}$ exists and equals Stab( $\left.q\right)$ for each global generic type $q$ of $G$. (Hence the cosets of $G^{00}$ in $G$ correspond to the translates of $\left.q.\right)$

Proof. Let $p$ be the type given by $f s g$. So for all sufficiently large $n$, " $x \in G_{n}$ " $\in p$. Likewise any definable subset $X$ of $p$ is in $G_{n}$ for sufficiently large $n$. So given a definable left generic set $X$, there is an $n$ such that " $x \in G_{n}$ " $\in p$ and $X \subseteq G_{n}$. So (as finitely many left translates of $X$ cover $G_{n}$ ) some left translate of $X$ is in $p$ and hence $X$ is in some left translate of $p$, so $X$ meets $M_{0}$. Likewise every left translate of $X$ meets $M_{0}$. Now fix $m$. Then for every $g \in G_{m}, g X$ meets $M_{0}$. By compactness there are $g_{1}, \ldots, g_{k} \in G\left(M_{0}\right)$ such that for every $g \in G_{m}, g X$ contains one of the $g_{i}$. But then for every $g \in G_{m}, g^{-1} \in X g_{1} \cup \ldots \cup X g_{k}$. As $G_{m}=G_{m}^{-1}$, we see that finitely many right translates of $X$ cover $G_{m}$. As $m$ was arbitrary, we conclude that $X$ is right generic. The rest of (i) follows by the same argumentation (noting that every right translate of $p^{-1}$ is finitely satisfiable in $M_{0}$ ).

(ii) follows from (i).

(iii) Again we may suppose that $X \subseteq G_{n}$ and $p(x) \models$ " $x \in G_{n}$ ". So some translate of $X$ is in $p$, so $X$ is in a translate of $p$, so $X_{1}$ or $X_{2}$ is in the same translate of $p$ and so is generic. 
At this point we see that the collection of nongeneric definable subsets of $G$ is an ideal. Call this ideal $\mathcal{I}$.

(iv) Suppose $H$ to be a subgroup of $G$ of bounded index which is $A$-invariant for some small set $A$. Then our given global generic type $p$ determines a coset of $H$ in $G$ and every other coset of $H$ in $G$ corresponds to a translate of $p$. So the number of translates of $p$ bounds the index of $H$ in $G$. Hence there is a smallest such $H$ (even as $A$ varies).

(v) requires a little finesse. First let $X$ be any definable subset of $G$. Let $\operatorname{Stab}_{\mathcal{I}}(X)$ be as in Section 4, namely $\{g \in G: g X \Delta X$ is nongeneric in $G\}$. So $\operatorname{Stab}_{\mathcal{I}}(X)$ is a subgroup of $G$ but on the face of it has no definability properties. But we DO know that $\operatorname{Stab}_{\mathcal{I}}(X)$ is invariant over the parameters defining $X$ and also has bounded index in $G$ (as generics meet $M_{0}$ ). Now fix a global generic type $q$. By what we have just said, together with (iv), $\bigcap\left\{\operatorname{Stab}_{\mathcal{I}}(X): X \in q\right\}=H$ say is a subgroup of bounded index invariant over some small set, and $H$ is clearly contained in $\operatorname{Stab}(q)$. But there is an $n$ such that $q(x) \models$ " $x \in G_{n}$ ", and therefore, as above, $G_{n}$ and every $G_{m}, m>n$, are generic in $G$.

Clearly $\operatorname{Stab}(q)$ is a subgroup of $G$ contained in $G_{n+1}$. Thus $H \subseteq G_{n+1}$. Now for $X \in q$, let $S t a b_{\mathcal{I}}^{n+1}(X)=\left\{g \in G_{n+1}: g X \Delta X\right.$ is nongeneric $\}$. But this is clearly type-definable (as we only have to say that finitely many translates of $g X \Delta X$ do not cover $\left.G_{n+2}\right)$. As $H=\bigcap\left\{S t a b_{\mathcal{I}}^{n+1}(X): X \in q\right\}$, it follows that $H$ is type-definable. So we have constructed a type-definable subgroup of $G$ of bounded index. By (iv) there is a smallest one, so $G^{00}$ exists. As in the earlier proof, $G^{00}$ must contain $\operatorname{Stab}(q)$. So $G^{00}=H=\operatorname{Stab}(q)$.

We can easily generalize Proposition 6.1 as well.

Proposition 7.4. Assume that $T$ has the NIP and $G=\bigcup_{n} G_{n}$ is an Ind-definable group (Ind-definable over $\emptyset$ ). Suppose that $G H A S$ a type-definable subgroup of bounded index. Then it has a smallest one, $G^{00}$, which is moreover normal and type-definable over $\emptyset$.

Proof. Note by assumption (**) that if $H$ is a type-definable subgroup of $G$, contained in $G_{n}$ say, then $G / H$ has bounded cardinality iff $G_{n} / H$ does.

By our assumptions, without loss of generality there is a type-definable subgroup $H$ of $G$ of bounded index, which is contained in $G_{0}$. The proof of Proposition 6.1 goes through word for word to give a type-definable subgroup $L_{0}$ of $G$ of bounded index which is smallest among those contained in $G_{0}$. Likewise for each $n$ there is a type-definable subgroup $L_{n}$ of $G$ which is smallest among those contained in $G_{n}$. But then $L_{n} \subseteq L_{0}$, so $L_{n}$ is contained in $G_{0}$, so $L_{n}=L_{0}$. Thus $L_{0}=G^{00}$. It is clearly normal and type-definable over $\emptyset$.

Lemma 7.5. Assume that $G$ is an Ind-definable group as above and that $G^{00}$ is a minimal type-definable subgroup of bounded index. Let $\pi: G \rightarrow G / G^{00}$ be the projection map and set $Y \subseteq G / G^{00}$ to be closed if and only if for every $n$, $\pi^{-1}(Y) \cap G_{n}$ is type-definable. Then these closed sets generate a locally compact topology on $G$, making it into a topological group.

The compact sets in $G / G^{00}$ are those closed $Y$ such that $\pi^{-1}(Y)$ is contained in $G_{n}$ for some $n$.

Proof. Left to the reader. 
Remark 7.6. In fact one can formulate the notion of a "type-definable" equivalence relation $E$ on an Ind-definable set $Y$, and assuming boundedly many classes, one can define the "logic topology" on $Y / E$ which will be locally compact. As we will only require the group case as in Lemma 7.5, we leave details of the general case to the reader.

Finally we generalize Proposition 6.2 to the Ind-definable setting. Recall first that a left Haar measure on a locally compact group $G$ is a left invariant Borel measure $\mu$ on $G$ such that $\mu(X)$ is finite for $X$ compact and positive for $X$ open (so it may take value $\infty$ on some Borel sets). A left Haar measure exists and is unique up to multiplication by a positive real.

Proposition 7.7. Let $G$ be an Ind-definable group with finitely satisfiable generics. Assume $T$ has NIP. Then there is a left invariant Keisler measure on $G$ which is moreover finitely satisfiable in some small model.

Proof. As in the proof of Proposition 6.2, we may assume $T$ to be countable. Let $\mathbf{m}$ be a right Haar measure on the locally compact group $G / G^{00}$. Let $p(x)$ be a global generic type extending $G^{00}$. Without loss of generality $G^{00}$ is contained in $G_{0}$.

We would like (as in the proof of Proposition 6.2) to define a left invariant Keisler measure $\mu_{p}$ on $G$ by stipulating that for any definable subset $X$ of $G$, $\mu_{p}(X)=\mathbf{m}\left(\left\{g / G^{00}: g X \in p\right\}\right)$.

So fix a definable subset $X$ of $G$. Assume $X \subseteq G_{n}$. As before, whether or not $g X$ is in $p$ depends only on $g / G^{00}$. So the main point is to see that $\left\{g / G^{00}: g X \in p\right\}$ is Borel and has finite $\mathcal{M}$-measure.

Note that if $g X \in p$, then $g \in G_{n+1}$ (as $g \in G_{0} \cdot G_{n}$ ) and so $g X \subseteq G_{n+2}$. We copy the proof of Proposition 6.2 but now defining $U$ to be $\left\{Y \cap G_{n+2}\left(M_{0}\right): Y \in p\right\}$ and concluding that $\left\{g / G^{00}: g X \in p\right\}$ is a Borel subset of the compact set $G_{n+1} / G^{00}$ and hence has finite $\mathbf{m}$-measure. So we can define $\mu_{p}$. Left invariance, finite additivity, and finite satisfiability in $M_{0}$ are proved as before.

We conclude this interlude with a result that appears at first sight close to the conjectures for compact groups, mentioned in the introduction.

Proposition 7.8. Let $\bar{M}$ be a saturated o-minimal structure (expansion of a real closed field) and $G$ a definably connected group definable in $\bar{M}$. Then there is a definably compact neighbourhood of the identity $G_{0}=G_{0}^{-1}$ such that putting $G_{n}=$ $G_{0} \ldots G_{0}$ and $G_{\infty}=\bigcup_{n} G_{n}$, then the Ind-definable group $G_{\infty}$ has a unique smallest type-definable subgroup of bounded index $G_{\infty}^{00}$ and the quotient $L=G_{\infty} / G_{\infty}^{00}$ with the "logic topology" is a connected Lie group of the same dimension as the o-minimal dimension of $G$.

Proof. We can identify some neighborhood of 1 in $G$ with a neighborhood of 0 in $R^{n}$; write $*$ for multiplication in $G$. The only possible linear approximation to $x * y$ is $x+y$, by associativity and the existence of differentiable inverse. So letting $|x|=\max \left|x_{i}\right|$, for any $C>0$, for all sufficiently small $e>0$, if $|x| \leq e$ and $|y| \leq e$, then

$$
|x * y-(x+y)| \leq C|(x, y)| \text {. }
$$

Take $C$ infinitesimal and then $e$ infinitesimal compared to it, and let $U=\{x:|x| \leq$ $e\}, H=\{x:|x| \leq(1 / n) e, n=1,2, \ldots\}$. Then by (1) it is clear that $H$ is a typedefinable normal subgroup. Let $G_{0}=U \cup U^{-1}$ in the sense of (**), so as to have it 
symmetric. Let $G_{\infty}$ be the Ind-definable group generated by $U$, or equivalently by $G_{0}$. Modulo $H, *$ agrees with + on $U$, indeed on $G_{\infty}$. In particular $G_{\infty} / H \simeq \mathbb{R}^{n}$.

It remains only to show that $H$ equals $G^{00}$ precisely, i.e. that $G_{\infty} / G_{\infty}^{00}$ cannot have dimension bigger than the $o$-minimal dimension of $G$. We postpone this to Section 10; see Corollary 10.10 .

However, note that the locally compact quotient we obtained is abelian; it is indeed a locally compact manifestation of the Lie algebra of $G$. We feel that the canonical compact quotient of a definably compact group $K$ reflects better the structure of $K$; for instance $K / K^{00}$ is nonabelian if $K$ is nonabelian. In the general case too, there should also be a locally compact quotient whose structure is close to that of $G$. We do not at the moment have a precise statement of this, either in the compact or in the locally compact cases.

Note that the adjoint action $G \times L \rightarrow L$ is definable, in the sense of Section 2 .

\section{Proof of the o-Minimal Conjectures}

We now use some of the preceding results to complete the proof of the conjectures on definably compact definable groups in o-minimal structures from 23 . In fact we will prove a bit more, namely that such groups have $f s g$ and therefore by Proposition 6.2 are definably amenable. Our main result (stated in the language of Definition 2.1) is

Theorem 8.1. Let $\bar{M}$ be a saturated o-minimal expansion of a real closed field. Let $G$ be a definably connected definably compact group definable in $\bar{M}$. Then the following hold.

(i) G has fsg.

(ii) There is a definable surjective homomorphism $\pi: G \rightarrow H$ from $G$ to a compact Lie group $H$ such that the Lie group dimension of $H$ equals the o-minimal dimension of $G$ and moreover such that any definable homomorphism from $G$ to a compact group factors through $\pi$.

Of course the $H$ in part (ii) of the theorem is precisely $G / G^{00}$ equipped with the logic topology. We know from [4] that $G^{00}$ exists and $G / G^{00}$ is, as a topological group, a compact connected Lie group. As discussed in [19, we may assume that $G$ is a definable closed subset of some $\bar{M}^{n}$ and that the group operation on $G$ is continuous with respect to the induced topology on $G$.

We will prove Theorem 8.1 by proving it when $G$ is commutative and when $G$ is "semisimple" and then use Proposition 4.5 among other things to conclude the general case. For the rest of this section $\bar{M}$ is a saturated $o$-minimal expansion of a real closed field.

Lemma 8.2. Theorem 8.1 is true when $G$ is commutative.

Proof. We use additive notation for $G$. We first prove (ii). $T$ being $o$-minimal has NIP. Also as $G$ is commutative, it is amenable, so in particular definably amenable. Also by [19] the family of nongeneric definable subsets of $G$ forms an ideal $\mathcal{I}$. We can apply Proposition 6.3 to conclude that $\operatorname{Stab}_{\mathcal{I}}(X)$ is a type-definable subgroup of $G$ of bounded index for any definable subset $X$ of $G$. It is explained in 19. how this implies (ii), but we briefly recall the argument. For each $n$, we can find a definable subset $X_{n}$ of $G$ such that the sets $X_{n}, X_{n}+c_{1}, \ldots, X_{n}+c_{r}$ form a partition of $G$, where $0, c_{1}, \ldots, c_{r}$ are the elements of order $n$ in $G$. Then $\operatorname{Stab}_{n g}\left(X_{n}\right)$ contains no 
$n$-torsion (except 0). So if we know that each $\operatorname{Stab}_{n g}(X)$ has bounded index, it will follow that $G^{00}$ is contained in every $\operatorname{Stab}_{n g}\left(X_{n}\right)$ and hence has no torsion. As $G^{00}$ is divisible (see [4), it follows that $G$ and $G / G^{00}$ have isomorphic torsion.

By a theorem of Edmundo and Otero (see [7]), the torsion of $G$ is isomorphic to the torsion of $\left(S^{1}\right)^{\operatorname{dim}(G)}$. Hence the compact commutative Lie group $G / G^{00}$ must also be $\left(S^{1}\right)^{\operatorname{dim}(G)}$. So (ii) is proved.

Now for (i). Let $\sim_{\mathcal{I}}$ be the equivalence relation: " $X \Delta Y$ is nongeneric" on definable subsets of $G$. By Proposition 6.3(i) there are only boundedly many definable subsets of $G$ up to $\sim_{I}$. (Note that this already proves that $G$ has a bounded number of generic types.) Thus there is a small model $M_{0}$ such that $G$ is defined over $M_{0}$ and for every generic definable subset $Y$ of $G$ there is an $M_{0}$-definable subset $X$ of $G$ such that $Y \sim_{I} X$. To prove that $G$ has $f s g$, it is clearly enough (given the existence of generic types) to prove that every generic definable subset $Y$ of $G$ meets $G\left(M_{0}\right)$. So let $Y \subseteq G$ be definable and generic.

Claim 1. There exists a definable subset $Y^{\prime} \subseteq Y$ which is closed (in $G$, so in $\bar{M}^{n}$ ) and still generic.

Proof. First, we may replace $Y$ by its interior. Now, for every $\epsilon>0$ we consider the set $Y_{\epsilon}$ of all $y \in Y$ whose distance from the frontier of $Y$ is greater than $\epsilon$ (in the sense of $\bar{M}^{n}$ ). Because the frontier of $Y$ is not generic, there is some $\epsilon>0$ for which $Y_{\epsilon}$ is generic, and we take it to be $Y^{\prime}$.

So we may assume $Y$ to be closed. Let $X$ be an $M_{0}$-definable subset of $G$ such that $Y \sim_{n g} X$. We may clearly assume $X$ to be closed (as $c l(X) \backslash X$ is nongeneric). Hence $X \cap Y$ is closed. Let $Z=X \backslash Y$.

Claim 2. The set of $M_{0}$-conjugates of $X \cap Y$ is finitely consistent.

Proof. Otherwise (as $X$ is $M_{0}$-definable) finitely many $M_{0}$-conjugates of $Z$ cover $X$. But $Z$ is nongeneric in $G$ as is any $M_{0}$-conjugate of $Z$. So $X$ is the union of finitely many nongenerics, while itself being generic. This is a contradiction.

By Claim 2 and Theorem 2.1 of [19] (which comes out of Dolich's work [5]), $X \cap Y$ meets $M_{0}$, as does $Y$. This completes the proof of (i) and of Lemma 8.2.

Let $G$ be definable in $\bar{M}$. We will say that $G$ has very good reduction if it is definably isomorphic, in $\bar{M}$, to a group $G_{1}$ with the following property: There is a sublanguage $L_{0}$ of the language $L$ of $\bar{M}$ which contains,$+ \cdot$ and there is an elementary substructure $M_{0}$ of $\bar{M} \mid L_{0}$ whose underlying set is $\mathbb{R}$ and such that $G_{1}$ is definable by an $L_{0}$-formula with parameters from $M_{0}$, i.e. from $\mathbb{R}$. (But note that $M_{0}$ need not be expandable to an elementary substructure of $\bar{M}$.)

Remark. This notion of very good reduction is related to, but not identical with, the algebraic-geometric notion in the case of saturated real closed fields and the natural valuation. In any case it is important to note that even if $R$ is a saturated real closed field, there will be definable groups, even real algebraic ones which do not have very good reduction in the model theoretic sense above. Indeed, as was shown in [21, if $R$ is a sufficiently saturated real closed field, then not all elliptic curves over $\operatorname{acl}(R)$ are definably isomorphic to each other (as groups). In fact this remains true even in an expansion of $R$ to a structure $R_{a n}$ elementarily equivalent to $\mathbb{R}_{a n}$. Now, in $\mathbb{R}_{a n}$ all definable compact abelian groups of fixed dimension (defined over 
$\mathbb{R})$ are definably isomorphic to each other; therefore, even in $R_{a n}$ not all elliptic curves over $\operatorname{acl}(R)$ have very good reduction.

Lemma 8.3. Theorem 8.1 holds when $G$ has very good reduction.

Proof. Part (ii) of the theorem is precisely Fact 4.1 of [19]. The fact that the nongeneric sets form an ideal was proved in [19], but this as well as the rest of (i) follows directly from Proposition 4.6 in the same paper (which itself depends on results of Berarducci and Otero [2]). More precisely (with above notation) Proposition 4.6 of [19] states among other things that if $X \subset G$ is definable (in $\bar{M}$ ), then $X$ is left generic iff right generic iff $X$ contains an open set which is $L_{0}$-definable over $M_{0}$. This on the one hand implies that there exists a complete generic type and on the other hand that if we pick $M_{1}$ to be any elementary substructure of $\bar{M}$ which contains $M_{0}$, then any generic definable subset of $X$ meets $M_{1}$. Thus $G$ has fsg.

Proof of Theorem 8.1. Let $G$ be an arbitrary definable, definably connected, definably compact group in $\bar{M}$. We prove the theorem by induction on $\operatorname{dim}(G)$. If $G$ is "semisimple", namely has no proper connected infinite definable normal commutative subgroup, then by [17, $G$ is an almost direct product of finitely many almost definably simple groups $G_{1}, \ldots, G_{k}$. ("Almost definably simple" means that the group is noncommutative and the quotient by some finite normal subgroup is definably simple.) Now by [18. (see the proof of $(2) \Rightarrow(3)$ in Theorem 5.1 there), any definably simple group is definably isomorphic to some semialgebraic group defined over $\mathbb{R}$. In particular, a definably simple group has very good reduction. It easily follows from Lemma 8.3 that Theorem 8.1 holds for a semisimple $G$.

Thus we may assume that $G$ has an infinite, definably connected normal commutative subgroup $N$. By Lemma 8.2, the theorem is true of $N$, so we may assume $N \neq G$. By induction, the theorem is true for $G / N$, so by Proposition 4.5, $G$ has fsg.

All that is left to do is to prove that the dimension of the compact Lie group $G / G^{00}$ equals the $o$-minimal dimension of $G$. Notice first that the image of $G^{00}$ under the projection onto $G / N$ is necessarily $(G / N)^{00}$ (on one hand this image contains $(G / N)^{00}$; on the other hand the pre-image of $(G / N)^{00}$ is of bounded index and therefore contains $\left.G^{00}\right)$. Thus, it suffices to show that $G^{00} \cap N=N^{00}$. By [4] it is enough to prove

Claim. $G^{00} \cap N$ is torsion-free.

Proof. Fix $n$. Let us first choose a definable subset $X$ of $N$ such that $N$ is the disjoint union of the translates of $X$ by the distinct $n$-torsion points $1, g_{1}, \ldots, g_{r}$ say of $N$. (As usual $X$ is obtained by considering the surjective endomorphism $\pi: x \rightarrow n x$ of $N$ with itself, which has finite kernel, and using the existence of definable Skolem functions.) Likewise, using definable Skolem functions, we can find a definable subset $D$ of $G$ which meets every coset of $N$ in $G$ in a unique point. It follows that the definable sets $X D, g_{1} X D, \ldots, g_{r} X D$ are disjoint and cover $G$. By Corollary 4.3, $G^{00}$ is contained in $\operatorname{Stab}_{\mathcal{I}}(X)$, and clearly the latter does not contain any of $g_{1}, \ldots, g_{r}$. As $n$ was arbitrary, it follows that $G^{00} \cap N$ is torsion-free. This completes the proof of Theorem 8.1 . 
By Proposition 6.2 we conclude also

Corollary 8.4. Let $G$ be a definably compact group definable in $\bar{M}$. Then $G$ is definably amenable. In fact there is a left invariant Keisler measure on $G$ which is finitely satisfiable in some small model.

Remark. 1. In the very last step of the above proof we showed that, under the given assumptions, $G^{00} \cap N=N^{00}$. This is not true in general, even if we assume that $G, G / N$ and $N$ all have NIP and $f s g$. Indeed, consider the group $G=\langle\mathbb{C},+\rangle \oplus S^{1}$ ( $S^{1}$ the circle group), with predicates for $S^{1}$ and all its semialgebraic subsets (but not for $\mathbb{C}$ !). We have $G^{00}=G$, but $\left(S^{1}\right)^{00}$ is nontrivial.

2. Our proof of Theorem 8.1 depends in a crucial manner on the result [7] describing the torsion in definably compact commutative groups, which itself relies on quite intricate tools from algebraic topology. It would be desirable to have a "direct" proof of the latter in the spirit of the current paper. In fact we do have a reasonably elementary proof of the existence of torsion points (in commutative definably compact definably connected groups), which we sketch here:

(i) Using definable compactness, find a definable $X \subset G$ such that both $X$ and its complement $X^{c}$ are generic (this can be done similarly to the proof of Claim 1 above).

(ii) It follows that $\operatorname{Stab}_{n g}(X) \neq G$, and thus (as we saw that $\operatorname{Stab}_{n g}(X)$ has bounded index), $G^{00} \neq G$.

(iii) Since $G / G^{00}$ is a compact connected commutative nontrivial Lie group (4]), it has torsion, and since $G^{00}$ is divisible ([4), $G$ itself has torsion.

3. Notice that if a definable $G$ in an $o$-minimal structure has $f s g$, then it necessarily implies that $G$ is definably compact. Indeed, if $G$ were not definably compact, then by [22, $G$ has a definable one-dimensional, ordered subgroup $H$. Let $D \subseteq G$ be a definable set containing one representative for each coset of $H$, and let $I=(0, \infty) \subseteq H$. Then $D \cdot I$ is nongeneric in $G$ and so is its complement, contradicting Proposition 4.2 .

4. The proof of the o-minimal group conjecture that we give here depends in the ambient real closed field in two different ways. Firstly, in order to ensure that our group can be embedded as a topological group into some $R^{n}$, see a discussion in [19. Secondly (and more substantially) the above count of torsion points by Edmundo and Otero was only carried out for expansions of real closed fields. The conjecture was proved separately for groups definable in ordered vector spaces over division rings (see [15], [8]).

\section{Compact domination}

The third author has mentioned in previous papers that the o-minimal conjectures (solved in the last section) have the heuristic content that the map $G \rightarrow G / G^{00}$ should be a kind of intrinsic "standard part map". It is reasonable to attempt to give some concrete mathematical meaning to this, namely to come up with a model theory of "standard-part-like" maps (in a tame context). So we introduce the notion of "compact domination". It is analogous to "stable domination" from 12 which was introduced with algebraically closed-valued fields as a central example. We relate compact domination to the existence and uniqueness (and smoothness) of suitable Keisler measures and prove that in the cases we understand well (very 
good reduction and dimension 1) definably compact groups in o-minimal structures are compactly dominated (by $G / G^{00}$ ).

We begin by working in a saturated model $\bar{M}$ of an arbitrary theory. When we say compact, we mean compact Hausdorff. $G$ denotes a definable (or even typedefinable) group. We use freely the notion from Section 2 of a definable map from $X$ to a compact space. Also we will assume that every Borel measure $\mu$ on a compact space $C$ that we discuss is regular. Note that Haar measure is regular.

Definition 9.1. (i) Suppose $X$ is type-definable, $\pi: X \rightarrow C$ is a definable surjective map from $X$ to a compact space $C$, and $\mu$ is a probability measure on $C$. We say that $X$ is compactly dominated by $(C, \mu, \pi)$ if for any definable (that is relatively definable with parameters) subset $Y$ of $X$ and for every $c \in C$ outside a set of $\mu$ measure zero, either $\pi^{-1}(c) \subseteq Y$ or $\pi^{-1}(c) \subseteq X \backslash Y$. Namely,

$$
\mu\left(\left\{c \in C: \pi^{-1}(c) \cap Y \neq \emptyset \text { and } \pi^{-1}(c) \cap(X \backslash Y) \neq \emptyset\right\}\right)=0 .
$$

(ii) Let $G$ be a type-definable group. We say that $G$ is compactly dominated as a group if $G$ is compactly dominated over by $(H, \mathbf{m}, \pi)$ where $H$ is a compact group, $\mathbf{m}$ is the unique normalized Haar measure on $H$ and $\pi$ is a group homomorphism.

Note that in (i) above the set $\left\{c \in C: \pi^{-1}(c) \cap Y \neq \emptyset\right.$ and $\left.\pi^{-1}(c) \cap(X \backslash Y) \neq \emptyset\right\}$ is a closed subset of $C$, hence measurable.

When we work with a definable group $G$, we always refer to compact domination in the group sense.

Question 9.2. To what extent does the definition of compact domination depend on the choice of "measure zero" as the notion of "smallness" in $C$ ?

It would be interesting to investigate other possibilities. Smallness notions based on Baire category or dimension are more natural since they depend only on the topology; but in the context of groups the Haar measure also depends only on the topology and group structure and connects naturally to the topics discussed in this paper. It would be nice if for groups these notions turned out to be equivalent.

Let $P$ be compactly dominated via $\pi: P \rightarrow C$, where $P$ and $\pi$ are (type-) defined over $\emptyset$. We will say " $\theta(x, b)$ holds for almost all $x \in P$ " if $\mu(\pi(\{x$ : $\neg \theta(x, b)\}))=0$. We can write $\left(d_{P} x\right) \theta(x, b)$ for this. Note that this gives a partial type: $\left\{b:\left(d_{P} x\right) \theta(x, b)\right\}$ is type-definable over $\emptyset$. Indeed let $\left\{W_{i}\right\}_{i \in I}$ be the set of all closed subsets of $C$ of positive measure; then $\pi^{-1}\left(W_{i}\right)=\bigcap_{j} W_{i j}$ for some definable sets $W_{i j}$. Now $\neg\left(d_{P} x\right) \theta(x, b)$ iff $\mu(\pi(\theta(x, b)))>0$ iff $\pi(\theta(x, b))$ contains a closed set $W_{i}$ of measure $>0$, iff for some $i, j, \pi(\neg \theta(x, b))$ contains $W_{i j}$. The case of Baire category is similar.

This is again in analogy with the stably dominated case, where one obtains definable types.

One could ask to what extent $C$ is determined by $P$. If $P$ is compactly dominated via $\pi_{i}: P \rightarrow C_{i}$, there exist continous maps $f_{1}: C_{1}^{\prime} \rightarrow C_{2}$ and $f_{2}: C_{2}^{\prime} \rightarrow C_{1}$, where $C_{i}^{\prime}$ is a large subset of $C_{i}$, such that $f_{1} \pi_{1}=f_{2}$ for all $x \in \pi_{1}^{-1}\left(C_{1}^{\prime}\right)$, and dually. However in general $f_{1}, f_{2}$ are not inverses of each other.

Proposition 9.3. Suppose $G$ is compactly dominated by $(H, \pi)$. Then

(i) $G$ has finitely satisfiable generics, and

(ii) $G^{00}$ exists and equals $\operatorname{Ker}(\pi)$. 
Proof. Let us assume that $G$ is compactly dominated (over $\emptyset$ say) by the data. We go through various claims which eventually yield (i) and (ii). $Y$ will denote a definable subset of $G$ and $\pi^{\prime}(Y)=\left\{h \in H: \pi^{-1}(h) \subseteq Y\right\}$.

Claim 1. $\pi^{\prime}(Y) \subseteq \pi(Y), \pi(Y)$ is closed and $\pi^{\prime}(Y)$ is open.

Proof. Clear.

Claim 2. $\mathbf{m}(\pi(Y))=\mathbf{m}\left(\pi^{\prime}(Y)\right)$.

Proof. Because by the definition of compact domination $\mathbf{m}\left(\pi(Y) \backslash \pi^{\prime}(Y)\right)=0$.

Claim 3. The following are equivalent:

(a) $Y$ is left (right) generic,

(b) $\pi(Y)$ is left (right) generic,

(c) $\mathbf{m}(\pi(Y))>0$,

(d) $\pi^{\prime}(Y)$ is nonempty.

Proof. (a) implies (b) implies (c) is clear. Suppose now that (c) holds. Then by Claim 2, $\mathbf{m}\left(\pi^{\prime}(Y)\right)>0$, so in particular (d) holds. Now assume (d). Then $Y$ contains a coset of $K e r \pi$, which is type-definable of bounded index, and hence $Y$ is left and right generic.

Claim 4. If $Y=Y_{1} \cup Y_{2}$ (where the $Y_{i}$ are definable) and $Y$ is generic, then $Y_{1}$ or $Y_{2}$ is generic.

Proof. By Claim 3, $\mathbf{m}\left(\pi^{\prime}(Y)\right)>0$, but the compact domination assumption implies that $\mathbf{m}\left(\pi^{\prime}(Y)\right)=\mathbf{m}\left(\pi^{\prime}\left(Y_{1}\right)\right)+\mathbf{m}\left(\pi^{\prime}\left(Y_{2}\right)\right)$, so again by Claim 3 we are done.

Let $M_{0}$ be an elementary substructure of $\bar{M}$ containing representatives of each coset of $G$ modulo $\operatorname{Ker}(\pi)$.

Claim 5. If $Y$ is generic in $G$, then $Y$ meets $M_{0}$.

Proof. By Claim 3, $Y$ contains a whole coset of $\operatorname{Ker}(\pi)$.

By Claim 4 there is a global generic type $p$ of $G$. Every translate of $p$ is also generic so by Claim 5 is finitely satisfiable in $M_{0}$. Thus $G$ has $f s g$, giving part (i).

Let $\mathcal{I}$ be the ideal of nongeneric definable sets (which exists by Claim 5.)

Claim 6. $\operatorname{Ker}(\pi) \subseteq \operatorname{Stab}_{\mathcal{I}}(Y)$.

Proof. Let $g \in \operatorname{Ker}(\pi)$. Then $\pi(Y \Delta g Y) \subseteq\left(\pi(Y) \backslash \pi^{\prime}(Y)\right) \cup\left(\pi(g Y) \backslash \pi^{\prime}(g Y)\right)$. By Claim 2 the latter has Haar measure 0; hence by Claim 3, $Y \Delta g Y$ is nongeneric.

By Corollary 4.3. $G^{00}$ exists and equals the intersection of all $\operatorname{Stab}_{\mathcal{I}}(Y)$. Since $\operatorname{ker} \pi$ has bounded index in $G$, by Claim $6, G^{00}$ equals $\operatorname{Ker}(\pi)$.

Note that it follows that if $G$ is $\emptyset$-definable and compactly dominated over some parameters, then it is compactly dominated over any model (as $G^{00}$ is type-definable over $\emptyset$ ). We now aim towards the appropriate analogue of "existence and uniqueness of Haar measure" for compactly dominated groups. We begin with a group-free version:

Proposition 9.4. Let $X$ be type-definable over $\emptyset$ and compactly dominated over $\emptyset$ by $(C, \mu, \pi)$. Then the following hold.

(i) There is a unique Keisler measure $\mu^{\prime}$ on $X$ with the property that $\mu(D)=$ $\mu^{\prime}\left(\pi^{-1}(D)\right)$ for any closed $D \subseteq C$.

(ii) The Keisler measure $\mu^{\prime}$ from (i) is smooth (over $\emptyset$ ). 
Proof. We first start with an explanation. When in (ii) we refer to $\mu^{\prime}\left(\pi^{-1}(D)\right)$, we identify $\mu^{\prime}$ with the canonical countably additive Borel measure extending it, as discussed in Section 2, just before Construction $\left(^{*}\right)$.

In any case, let us first show the existence of $\mu^{\prime}$ : For $Y$ a (relatively) definable subset of $X$, put $\mu^{\prime}(Y)=\mu(\pi(Y))$. Note that $\mu^{\prime}$ DOES satisfy the condition in (i): for if $D \subseteq C$ is closed and $Y=\pi^{-1}(D)$, then $Y$ is type-definable so equals $\bigcap_{i} Y_{i}$ where $Y_{i}$ are (relatively) definable subsets of $X$. Let $D_{i}=\pi\left(Y_{i}\right)$. Then $D_{i}$ is closed in $C$ and $\bigcap_{i} D_{i}=D$. We may assume that the family $\left(Y_{i}\right)_{i}$ is closed under finite intersections. It follows (by our assumption of regularity of $\mu$ ) that $\mu(D)=\inf \left\{\mu\left(D_{i}\right): i \in I\right\}=\inf \left\{\mu^{\prime}\left(Y_{i}\right): i \in I\right\}=\mu^{\prime}(Y)$.

We must check finite additivity of $\mu^{\prime}$. But if $Y_{1}, Y_{2}$ are disjoint definable subsets of $X$, then (by compact domination) $\mu\left(\pi\left(Y_{1}\right) \cap \pi\left(Y_{2}\right)\right)=0$; hence $\mu^{\prime}\left(Y_{1} \cup Y_{2}\right)=$ $\mu^{\prime}\left(Y_{1}\right)+\mu^{\prime}\left(Y_{2}\right)$.

Now for uniqueness: Suppose $\mu^{\prime \prime}$ is another Keisler measure on $X$ such that $\mu(D)=\mu^{\prime \prime}\left(\pi^{-1}(D)\right)$ for any closed $D \subseteq C$. Let $Y$ be an arbitrary definable subset of $X$. Then, since $\pi^{-1} \pi^{\prime}(Y) \subseteq Y \subseteq \pi^{-1} \pi(Y)$, we have

$$
\mu\left(\pi^{\prime}(Y)\right)=\mu^{\prime \prime}\left(\pi^{-1} \pi^{\prime}(Y)\right) \leq \mu^{\prime \prime}(Y) \leq \mu^{\prime \prime}\left(\pi^{-1} \pi(Y)\right)=\mu(\pi(Y)) .
$$

But $\mu\left(\pi^{\prime}(Y)\right)=\mu(\pi(Y))$; hence $\mu^{\prime}(Y)=\mu^{\prime \prime}(Y)$. So we have proved (i).

Recall that the smoothness of $\mu^{\prime}$ over $\emptyset$ means by definition that $\mu^{\prime} \mid \emptyset$ has precisely one extension to a Keisler measure on (all definable subsets of) $X$. However, since $\mu^{\prime} \mid \emptyset$ satisfies the assumptions of (i), it follows that it has a unique extension.

Theorem 9.5. Suppose $G$ is compactly dominated. Then $G$ has a unique left invariant Keisler measure, which is moreover right invariant and smooth.

Proof. Let $\pi: G \rightarrow H=G / G^{00}$. As before $\mathbf{m}$ denotes the Haar measure on $H$.

Let $\mu^{\prime}$ be as in Proposition 9.4 and its proof, namely for definable $X \subseteq G, \mu^{\prime}(X)$ is by definition $\mathbf{m}(\pi(X))$. Note that $\mu^{\prime}$ will be both left and right invariant, as $\mathbf{m}$ is. By Proposition 9.4, $\mu^{\prime}$ is also smooth.

Now suppose $\mu^{\prime \prime}$ is another left invariant Keisler measure on $G$. Let $M_{0}$ be a model over which $\pi$ is definable. By [13, $\mu^{\prime \prime} \mid M_{0}$ extends uniquely to a countably additive measure on the $\sigma$-algebra of subsets of $G$ generated by the $M_{0}$-type-definable sets. We still call this $\mu^{\prime \prime} \mid M_{0}$ and note that it is left invariant. But then $\mu^{\prime \prime} \mid M_{0}$ induces a left invariant countably additive measure on $H$ : namely for $B$ a Borel subset of $H$, define its measure to be $\mu^{\prime \prime}\left(\pi^{-1}(B)\right)$. By uniqueness of Haar measure, this latter measure has to agree with $\mathbf{m}$. Hence we have shown that $\mathbf{m}(C)=\mu^{\prime \prime}\left(\pi^{-1}(C)\right)$. By Proposition 9.4(i), $\mu^{\prime \prime}=\mu^{\prime}$. This completes the proof.

\section{0. o-MINIMALITY AND COMPACT DOMINATION}

Let $\bar{M}$ denote now a saturated $o$-minimal expansion of an ordered divisible group $R$.

Beraducci and Otero, in their paper 2, prove in effect (for o-minimal expansions of real closed fields) that the unit $n$-cube $I^{n}$ in $\bar{M}$ is compactly dominated, with respect to the standard part map to $I^{n}(\mathbb{R})$ equipped with Lebesgue measure. This is not stated explicitly in their paper but follows from it. In any case we give below another proof of this fact (omitting the real closed field assumption), using the following beautiful theorem of Baisalov and Poizat (recall that a weakly ominimal structure is an ordered structure in which every definable subset of the linear ordering is a finite union of convex sets): 
Theorem ([1]). If the saturated o-minimal structure $\bar{M}$ is expanded by any number of convex subsets of $\bar{M}$, then the resulting structure is weakly o-minimal.

Some notation: We let $\mathbb{R}$ denote a fixed copy of the reals, which we may assume is a subgroup of $R$ (in particular, we have a copy of $\mathbb{Q}$ in $R$ ). Let Fin denote the set of finite elements of $R$ (i.e. absolute value less than $n$ for some $n \in \mathbb{N}$ ) and Inf the set of infinitesimals of $R$ (absolute value $<1 / n$ for all $n \in \mathbb{N}$ ). Let $\pi$ denote the "standard part map" from Fin onto Fin/Inf. Since Fin/Inf is archimedean (and $\bar{M}$ saturated), we can identify Fin/Inf with $\mathbb{R}$.

Let $\langle\bar{M}$, Fin, Inf $\rangle$ be the structure $\bar{M}$ equipped with unary predicates for Fin and Inf. Then the quotient group Fin/Inf is interpretable in it, and $\pi$ induces a canonical bijection $i:$ Fin $/$ Inf $\rightarrow \mathbb{R}$.

Definition 10.1. By $\mathbb{R}_{\text {ind }}$ (standing for " $\mathbb{R}$ with the induced structure") we mean the structure whose universe is $\mathbb{R}$ and whose relations are precisely the images under $i$ of subsets of $(\text { Fin/Inf })^{n}$ which are definable (with parameters) in $(\bar{M}$, Fin, Inf $)$.

Lemma 10.2. $\mathbb{R}_{\text {ind }}$ is o-minimal (in fact is an o-minimal expansion of the ordered group of $\mathbb{R})$.

Proof. It is clear that $<$ and the graphs of + and $\cdot$ are among the basic relations on $\mathbb{R}_{\text {ind }}$.

By [1] the structure $\langle\bar{M}$, Fin, Inf $\rangle$ is weakly o-minimal. Let $X \subseteq \mathbb{R}$ be definable in $\mathbb{R}_{\text {ind }}$. Then clearly $\pi^{-1}(X)$ is definable in $\langle\bar{M}$, Fin, Inf $\rangle$, so it is a finite union of convex sets. So $X$ has finitely many connected components. Thus $\mathbb{R}_{\text {ind }}$ is ominimal.

Lemma 10.3. Let $X \subset$ Fin $^{n}$ be definable in $\bar{M}$ with $\operatorname{dim}(X)<n$. Then $\operatorname{dim}(\pi(X))$ $<n$ (in the o-minimal structure $\mathbb{R}_{\text {ind }}$ ).

Proof. The proof is by induction on $n$ and is immediate for $n=1$. For an arbitrary $n$, we may assume by cell decomposition that $X$ is the graph of a continuous definable function $f: C \rightarrow R$, where $C$ is a definable open set in $R^{n-1}$. By $o-$ minimality of $\mathbb{R}_{\text {ind }}$, if $\operatorname{dim}(\pi(X))=n$, then it must contain the closure of a subset $U \times\left(q_{1}, q_{2}\right)$, for $U$ an open rectangular box of rational coordinates (which we may assume is contained in $C$ ) and $q_{1}, q_{2} \in \mathbb{Q}$.

Consider an arbitrary $x \in U(R)$ and $r$ a rational number in $\left(q_{1}, q_{2}\right)$. By assumptions, there exist $x_{1}, x_{2}$ infinitesimally close to $x$ such that $f\left(x_{1}\right), f\left(x_{2}\right)$ are infinitesimally close to $q_{1}, q_{2}$, respectively. But then, by continuity, there exists an $x^{\prime}$ infinitesimally close to $x$ such that $f\left(x^{\prime}\right)=r$. It follows that $\pi(\{x \in U(R)$ : $f(x)=r\})=U$, which by induction implies that the set $\{x \in U(R): f(x)=r\}$ has an interior in $R^{n-1}$. This can be done for any rational $r \in\left(q_{1}, q_{2}\right)$, a contradiction.

Theorem 10.4. Let $I^{n}$ be the unit $n$-cube in $R^{n}, \pi$ the standard part map from $I^{n}$ to $I^{n}(\mathbb{R})$, and $\mu$ the Lebesgue measure on $I^{n}(\mathbb{R})$. Then $I^{n}$ is compactly dominated (in $\bar{M})$ by $\left(I^{n}(\mathbb{R}), \mu, \pi\right)$.

Proof. Let $X \subseteq I^{n}$ be definable in $\bar{M}$. Let $Y$ be the frontier of $X$ (the set of $x$ such that every neighbourhood of $x$ contains points both in $X$ and not in $X$ ). Then $\operatorname{dim}(Y)<n$. So $\operatorname{dim}(\pi(Y))<n$ by Lemma 10.3. As $\pi(Y)$ is definable in the $o$-minimal structure $\mathbb{R}_{\text {ind }}$, it follows that the Lebesgue measure of $\pi(Y)$ is 0 . Note also that $\pi(Y)$ is closed. For $c \in I^{n}(\mathbb{R})$, the type-definable set $\pi^{-1}(c)$ is definably 
connected (cannot be written as the union of two relatively open relatively definable subsets). So for $c \in I^{n}(\mathbb{R}) \backslash \pi(Y)$, either $\pi^{-1}(c)$ is contained in $X$ or it is contained in the complement of $X$. This proves compact domination.

We are now in a position to state a rather finer version of the conjectures from 23. As before $\pi$ denotes the homomorphism from $G$ onto $G / G^{00}$ and $\mathbf{m}$ denotes Haar measure on $G / G^{00}$.

Compact Domination Conjecture. Any definably compact group $G$ (definable in a saturated $o$-minimal expansion of a real closed field) is compactly dominated (by the compact Lie group $G / G^{00}$, with its Haar measure $\mathbf{m}$ ).

Note that, by Proposition 9.3, if $G$ (definably compact in a saturated $o$-minimal expansion of a real closed field) is compactly dominated by $H$, then $H$ has to coincide with the compact Lie group $G / G^{00}$.

The following lemma allows us to reduce the Compact Domination Conjecture to a simpler statement.

Lemma 10.5. Suppose $G$ is definably compact with $\operatorname{dim}(G)=n$, and suppose that whenever $Y \subseteq G$ is definable and $\operatorname{dim}(Y)<n$, then $\boldsymbol{m}(\pi(Y))=0$. Then $G$ is compactly dominated by $G / G^{00}$.

Proof. Note that $G$ here is equipped with its "definable topology". We make use of a key result from [4] which says that $G^{00}$ and each translate of it are definably connected. It follows that if $X \subseteq G$ is definable and if $Y$ is the frontier of $X$ in $G$ (which has dimension $<n$ ), then for all $c \notin \pi(Y), \pi^{-1}(c)$ is either contained in $X$ or disjoint from $X$. Now, just as in the proof of Theorem 10.4, we obtain compact domination.

The above conjecture, if proven true, will resolve an intriguing open problem regarding the connection between generic sets and torsion points.

Proposition 10.6. Assume that $G$ is a definable abelian group in $\bar{M}$ and that $G$ is compactly dominated by $G / G^{00}$ (with its Haar measure). Then every definable generic subset of $G$ contains a torsion point. In particular, if $X \subseteq G$ is generic, then there are finitely many torsion points $g_{1}, \ldots, g_{k}$ such that $G=\bigcup_{i} g_{i} X$.

Proof. If $X \subseteq G$ is generic, then, by Claims 1 and 3 in the proof of Proposition 9.3. $\pi^{\prime}(X)=\left\{g / G^{00}: g G^{00} \subseteq X\right\}$ is open in $G / G^{00}$ and therefore contains a torsion point. Since $G^{00}$ is divisible and torsion-free, the coset $g G^{00}$ and therefore $X$ contain a torsion point. The rest follows easily.

There is very little we currently know about the consequences of the above proposition. Indeed, we do not even know that every large set (namely, the complement of a definable subset of $G$ of small dimension) contains a torsion point.

Theorem 10.7. Let $G$ be a definably compact group definable in an o-minimal $\bar{M}$. Then $G$ is compactly dominated in either of the cases

(i) $\bar{M}$ expands a real closed field and $G$ has very good reduction,

(ii) $\operatorname{dim}(G)=1$. 
Proof. Case (i). We assume that there is a sublanguage $L_{0}$ of $L$ such that $G$ is defined in $L_{0}$ over the elementary substructure $M_{0}=\langle\mathbb{R},+,<, \ldots\rangle$ of $\bar{M} \mid L_{0}$. Assume $\operatorname{dim}(G)=n$. Then $G$ has a covering by finitely many charts $U_{1}, \ldots, U_{r}$, each of which is definably homeomorphic via some $f_{i}$ to an open definable subset $V_{i}$ of $I^{n}$ (all definable in $L_{0}$ over $M_{0}$ ). Let $\mathbb{R}_{i n d}$ be as above. As was pointed out earlier, $G^{00}$ is exactly the collection of all elements in $G$ that are infinitesimally close to $e$. Thus we identify $G / G^{00}$ with $G\left(\mathbb{R}_{i n d}\right)$. Suppose $Y \subseteq G$ is definable with $\operatorname{dim}(Y)<n$. Then working in the charts and using Proposition 10.3 , we see that $\operatorname{dim}(\pi(Y))<n$ in the o-minimal structure $\mathbb{R}_{\text {ind }}$. Then clearly $\mathbf{m}(\pi(Y))=0$. (For example, working in the charts, the Lebesgue measure of $\pi(Y)=0$, so the Haar measure must be 0 too.) Now apply Lemma 10.5.

Case (ii). If $\operatorname{dim}(G)=1$, then any definable subset $Y$ of $G$ of dimension $<1$ is finite, so $\pi(Y)$ is finite too and hence has Haar measure 0. Again apply Lemma 10.5 .

Corollary 10.8. Suppose $G$ is as in Theorem 10.7. Then there is a unique invariant Keisler measure on $G$, which is moreover smooth.

Proof. By Theorems 9.5 and 10.7 .

Finally we return to the promised completion of the proof of Proposition 7.8 , this time as an illustration of the Compact Domination Conjecture. Actually the dominating group is locally compact in this case; the modification of the definition is evident. We show initially that $G_{\infty}$ is (locally) compactly dominated via $G_{\infty} \rightarrow$ $G_{\infty} / H$; as a bi-product, this gives $H=G_{\infty}^{00}$.

Proposition 10.9. Let $G, H$ be as in Proposition 17.8. Then $G_{\infty}$ is (locally) compactly dominated via $G_{\infty} \rightarrow G_{\infty} / H$.

Proof. Let $U(y)=\{x:|x| \leq y\}$. So $U=U(e)$. Let $\widetilde{G}=\bigcup_{N \in \mathbb{N}} U(N e)$. By (1) of Proposition 7.8. $G_{\infty} \subseteq \widetilde{G}$, and $*,+$ coincide on $\widetilde{G}$ up to $H$. In fact $\widetilde{G}=G_{\infty}$, since $\widetilde{G} / H=\mathbb{R}^{n}$ and $U / H$ contains an open neighborhood of 0 in $\mathbb{R}^{n}$.

Since $*,+$ coincide on $\widetilde{G}$ up to $H$, the proposition reduces to the case $G=$ $\left(R^{n},+\right)$, where $(R,+)$ is the underlying additive group of the o-minimal structure. In this case, add predicates for both $\{x:(\exists N \in \mathbb{N})|x| \leq N e\}$ and for $\{x:(\forall N \in$ $\mathbb{N})|x|<e / N\}$, obtain weak-o-minimality of their quotient by [1], and proceed as in the proof of Theorem 10.4.

Corollary 10.10. $G_{\infty}^{00}=H$

Proof. A generic set has generic image in $G_{\infty} / H$, hence contains a nonsmall subset of $G_{\infty} / H$; hence the pullback contains at least one full coset of $H$. Since $G_{\infty} / H$ is bounded, $G_{\infty}^{00}=H$.

\section{ACKNOWLEDGMENTS}

Some of the work presented here was done while the authors were at the Isaac Newton Institute, Cambridge, for the spring 2005 Model Theory program. We would like to thank both the Newton Institute and the organizers of the program for their hospitality, ideal conditions and financial support. In addition to the referee, 
several other individuals and research groups have passed on to us comments on an earlier version of the paper as well as helpful suggestions. So we would also like to thank Alessandro Berarducci, Margarita Otero, and Lou van den Dries and participants in the UIUC model theory seminar.

\section{REFERENCES}

[1] Y. Baisalov and B. Poizat, Paires de structures o-minimales, JSL 63 (1998), 570-578. MR1627306 (99m:03063)

[2] A. Berarducci and M. Otero, An additive measure in o-minimal expansions of fields, Quarterly Journal of Mathematics 55 (2004), 411-419. MR2104681 (2005k:03087)

[3] A. Berarducci and M. Otero, Intersection Theory for o-minimal structures, APAL 107 (2001), 87-119, Lemma 10.5. MR:1807841 (2001m:03074)

[4] A. Berarducci, M. Otero, Y. Peterzil, and A. Pillay, A descending chain condition for groups in o-minimal structures, APAL 134 (2005), 303-313. MR2139910 (2006a:03052)

[5] A. Dolich, Forking and independence in o-minimal theories, JSL 69 (2004), 215-240. MR2039358 (2005g:03050)

[6] M. Edmundo, Locally definable groups in o-minimal structures, J. of Algebra 301 (2006), 194-223. MR2230327

[7] M. Edmundo and M. Otero, Definably compact abelian groups, Journal of Math. Logic, 4 (2004), 163-180. MR2114966 (2005m:03073)

[8] P. Eleftheriou, Ph.D. thesis, U. of Notre Dame.

[9] Euclid, Elements, Book V, tr. T.L. Heath.

[10] R. Grossberg, J. Iovino, and O. Lessmann, A primer of simple theories, Archive Math. Logic 41 (2002), 541-580. MR1923196 (2003f:03043)

[11] E. Hewitt and K. Ross, Abstract harmonic analysis. Vol. I, Grundlehren der Mathematischen Wissenschaften [Fundamental Principles of Mathematical Sciences], vol. 115, 2, SpringerVerlag, Berlin, 1979. MR551496 (81k:43001)

[12] E. Hrushovski, Valued fields, metastable groups, draft, 2004.

[13] H. J. Keisler, Measures and Forking, Annals of Pure and Applied Logic 43 (1987), 119-169. MR890599 (88i:03052)

[14] L. Newelski and M. Petrykowski, Weak generic types and coverings of groups, Fund. Math. 191 (2006), 201-225.

[15] A. Onshuus, Groups definable in $(\mathbb{Z},+,<)$, preprint, 2005.

[16] A. Onshuus and A. Pillay, Definable groups and $p$-adic Lie groups, preprint, 2005.

[17] Y. Peterzil, A. Pillay and S. Starchenko, Definably simple groups in o-minimal structures, Trans. AMS, 352 (2000), 4397-4419. MR1707202(2001b:03036)

[18] Y. Peterzil, A. Pillay and S. Starchenko, Linear groups definable in o-minimal structures, J. of Algebra 247 (2002), 1-23. MR1873380 (2002i:03043)

[19] Y. Peterzil and A. Pillay, Generic sets in definably compact groups, Fund. Math. 193 (2007), 153-170.

[20] Y. Peterzil and S. Starchenko, Definable homomorphisms of abelian groups in o-minimal structures, Annals of Pure and Applied Logic 101 (2000), 1-27. MR.1729742 (2000m:03090)

[21] Y. Peterzil and S. Starchenko, Uniform definability of the Weierstrass $\wp$-functions and generalized tori of dimension one, Selecta Mathematica, New Series 10 (2004), 525-550. MR2134454 (2006d:03063)

[22] Y. Peterzil and C. Steinhorn, Definable compactness and definable subgroups of $o$-minimal groups, J. London Math. Soc 59 (1999), 769-786. MR.1709079 (2000i:03055)

[23] A. Pillay, Type-definability, compact Lie groups, and o-minimality, J. Math. Logic 4 (2004), 147-162. MR2114965 (2006d:03064)

[24] A. Pillay, Geometric Stability Theory, OUP, 1996. MR.1429864 (98a:03049)

[25] B. Poizat, A Course in Model Theory, Springer-Verlag, 2000. MR.1757487 (2001a:03072)

[26] S. Shelah, Classification theory for elementary classes with the dependence property - a modest beginning, Sci. Japonicae 59 (2004), 265-316. MR 2062198 (2005m:03063)

[27] S. Shelah, Minimal bounded index subgroup for dependent theories, to appear in Proceedings AMS. 
Hebrew University of Jerusalem, Department of Mathematics, Jerusalem, Israel

University of Haifa, Department of Mathematics and Computer Science, Haifa, IsRAEL

University of Illinois, Department of Mathematics, Altgeld Hall, 1409 W Green Street Urbana, IL 61801, and University of Leeds, School of Mathematics, Leeds, LS2 9JT ENGLAND 